Table A1. Geographic information (latitude, longitude, elevation above sea level) for CASTNet sites used in our analysis. Sites determined to be regionally-representative are indicated in bold.

\begin{tabular}{|c|c|c|c|c|c|c|}
\hline Region & Site ID & Station Name & State & $\begin{array}{l}\text { Latitude } \\
\text { ( N) }\end{array}$ & $\begin{array}{c}\text { Longitude } \\
\text { ( W) }\end{array}$ & $\begin{array}{c}\text { Elevation } \\
\text { (m ASL) }\end{array}$ \\
\hline \multirow{4}{*}{ Northwest } & $\mathrm{NCS}$ & North Cascades NP & WA & 48.5 & 121.4 & 109 \\
\hline & OLY & Olympic NP & WA & 48.1 & 123.4 & 125 \\
\hline & MOR & Mount Rainier NP & WA & 46.8 & 122.1 & 421 \\
\hline & GLR & Glacier NP & MT & 48.5 & 114.0 & 976 \\
\hline \multirow{5}{*}{ California } & LAV & Lassen Volcanic NP & CA & 40.5 & 121.6 & 1756 \\
\hline & DEV & Death Valley NM & CA & 36.5 & 116.8 & 125 \\
\hline & $\mathrm{PIN}$ & Pinnacles NM & CA & 36.5 & 121.2 & 335 \\
\hline & JOT & Joshua Tree NM & CA & 34.1 & 116.4 & 1244 \\
\hline & YOS & Yosemite NP - Turtleback Dome & $\mathrm{CA}$ & 37.7 & 119.7 & 1605 \\
\hline \multirow{10}{*}{ Mountain West } & CNT & Centennial & WY & 41.4 & 106.2 & 3178 \\
\hline & GTH & Gothic & $\mathrm{CO}$ & 39.0 & 107.0 & 2926 \\
\hline & GRB & Great Basin NP & NV & 39.0 & 114.2 & 2060 \\
\hline & MEV & Mesa Verde NP & $\mathrm{CO}$ & 37.2 & 108.5 & 2165 \\
\hline & PND & Pinedale & WY & 42.9 & 109.8 & 2388 \\
\hline & ROM & Rocky Mountain NP & $\mathrm{CO}$ & 40.3 & 105.5 & 2743 \\
\hline & YEL & Yellowstone NP & WY & 44.6 & 110.4 & 2469 \\
\hline & GRC & Grand Canyon NP & $A Z$ & 36.1 & 112.2 & 2073 \\
\hline & CAN & Canyonlands NP & UT & 38.5 & 109.8 & 1814 \\
\hline & $\mathrm{CHA}$ & Chiricahua NM & AZ & 32.0 & 109.4 & 1570 \\
\hline \multirow{7}{*}{ Plains } & CAD & Caddo Valley & AR & 34.2 & 93.1 & 71 \\
\hline & STK & Stockton & IL & 42.3 & 90.0 & 274 \\
\hline & VOY & Voyageurs NP & $\mathrm{MN}$ & 48.4 & 92.8 & 427 \\
\hline & ALH & Alhambra & IL & 38.9 & 89.6 & 164 \\
\hline & BVL & Bondville & IL & 40.1 & 88.4 & 212 \\
\hline & PRK & Perkinstown & Wl & 45.2 & 90.6 & 472 \\
\hline & $\mathrm{VIN}$ & Vincennes & $\mathbb{N}$ & 38.7 & 87.5 & 134 \\
\hline \multirow{13}{*}{ Great Lakes } & ANA & Ann Arbor & $\mathrm{Ml}$ & 42.4 & 83.9 & 267 \\
\hline & $\operatorname{HOX}$ & Hoxeyville & Ml & 44.2 & 85.7 & 305 \\
\hline & UVL & Unionville & MI & 43.6 & 83.4 & 201 \\
\hline & SAL & Salamonie Reservoir & IN & 40.8 & 85.7 & 250 \\
\hline & DCP & Deer Creek & $\mathrm{OH}$ & 39.6 & 83.3 & 267 \\
\hline & LYK & Lykens & $\mathrm{OH}$ & 40.9 & 83.0 & 303 \\
\hline & OXF & Oxford & $\mathrm{OH}$ & 39.5 & 84.7 & 284 \\
\hline & QAK & Quaker City & $\mathrm{OH}$ & 39.9 & 81.3 & 372 \\
\hline & KEF & Kane Exp. Forest & $\mathrm{PA}$ & 41.6 & 78.8 & 622 \\
\hline & LRL & Laurel Hill & $\mathrm{PA}$ & 40.0 & 79.3 & 615 \\
\hline & MKG & M.K. Goddard & PA & 41.4 & 80.1 & 384 \\
\hline & CDR & Cedar Creek & W & 38.9 & 80.8 & 234 \\
\hline & PAR & Parsons & W & 39.1 & 79.7 & 510 \\
\hline \multirow{5}{*}{ Northeast } & WSP & Washington Crossing & NJ & 40.3 & 74.9 & 61 \\
\hline & ARE & Arendtsville & PA & 39.9 & 77.3 & 269 \\
\hline & PSU & Penn State & PA & 40.7 & 77.9 & 378 \\
\hline & CTH & Connecticut Hill & NY & 42.4 & 76.7 & 501 \\
\hline & $\mathrm{ABT}$ & Abington & CT & 41.8 & 72.0 & 209 \\
\hline \multirow{4}{*}{ Far Northeast } & WST & Woodstock & $\mathrm{NH}$ & 43.9 & 71.7 & 258 \\
\hline & $\mathrm{ACA}$ & Acadia NP & ME & 44.4 & 68.3 & 152 \\
\hline & ASH & Ashland & ME & 46.6 & 68.4 & 235 \\
\hline & HOW & Howland & ME & 45.2 & 68.7 & 69 \\
\hline \multirow{18}{*}{ Southeast } & BEL & Beltsville & MD & 39.0 & 76.8 & 46 \\
\hline & BWR & Blackwater NWR & MD & 38.4 & 76.1 & 4 \\
\hline & VPI & Horton Station & VA & 37.3 & 80.6 & 920 \\
\hline & PED & Prince Edward & VA & 37.2 & 78.3 & 150 \\
\hline & $\mathrm{SHN}$ & Shenandoah NP - Big Meadows & VA & 38.5 & 78.4 & 1073 \\
\hline & $\mathrm{CDZ}$ & Cadiz & KY & 36.8 & 87.8 & 189 \\
\hline & CKT & Crockett & KY & 37.9 & 83.1 & 455 \\
\hline & MCK & Mackville & KY & 37.7 & 85.0 & 353 \\
\hline & ESP & Edgar Evins & TN & 36.0 & 85.7 & 302 \\
\hline & GRS & Great Smoky NP - Look Rock & $\mathrm{TN}$ & 35.6 & 83.9 & 793 \\
\hline & SPD & Speedwell & TN & 36.5 & 83.8 & 361 \\
\hline & COW & Coweeta & NC & 35.1 & 83.4 & 686 \\
\hline & PNF & Cranberry & NC & 36.1 & 82.0 & 1219 \\
\hline & $\mathrm{BFT}$ & Beaufort & NC & 34.9 & 76.6 & 2 \\
\hline & CND & Candor & NC & 35.3 & 79.8 & 198 \\
\hline & SND & Sand Mountain & $\mathrm{AL}$ & 34.3 & 86.0 & 352 \\
\hline & $\mathrm{CVL}$ & Coffeeville & MS & 34.0 & 89.8 & 134 \\
\hline & GAS & Georgia Station & $\mathrm{GA}$ & 33.2 & 84.4 & 270 \\
\hline \multirow{2}{*}{ Florida / Gulf } & EVE & Everglades NP & $\mathrm{FL}$ & 25.4 & 80.7 & 2 \\
\hline & SUM & Sumatra & $\mathrm{FL}$ & 30.1 & 85.0 & 14 \\
\hline
\end{tabular}



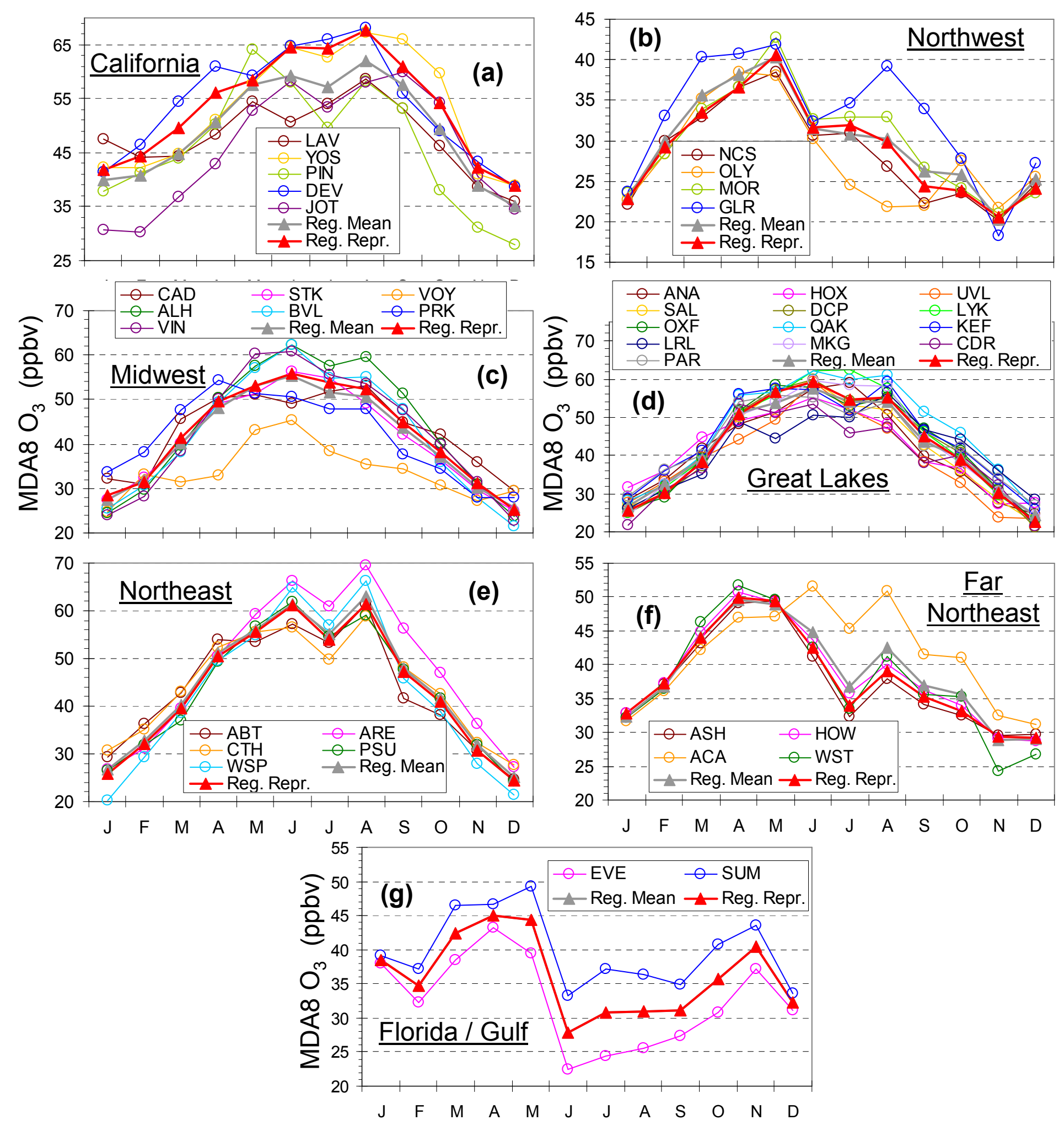

Figure A1. As in Fig. 2, but for the other seven regions. The regionally-representative sites (mean shown as solid red triangles) are: Northwest - MOR, NCS; California - DEV, YOS; Plains - BVL, CAD, STK; Great Lakes - DCP, MKG, OXF, SAL; Northeast CTH, PSU, WSP; Far Northeast - ASH, HOW; Florida / Gulf - EVE, SUM. Note the range of magnitudes on the $y$-axes. 

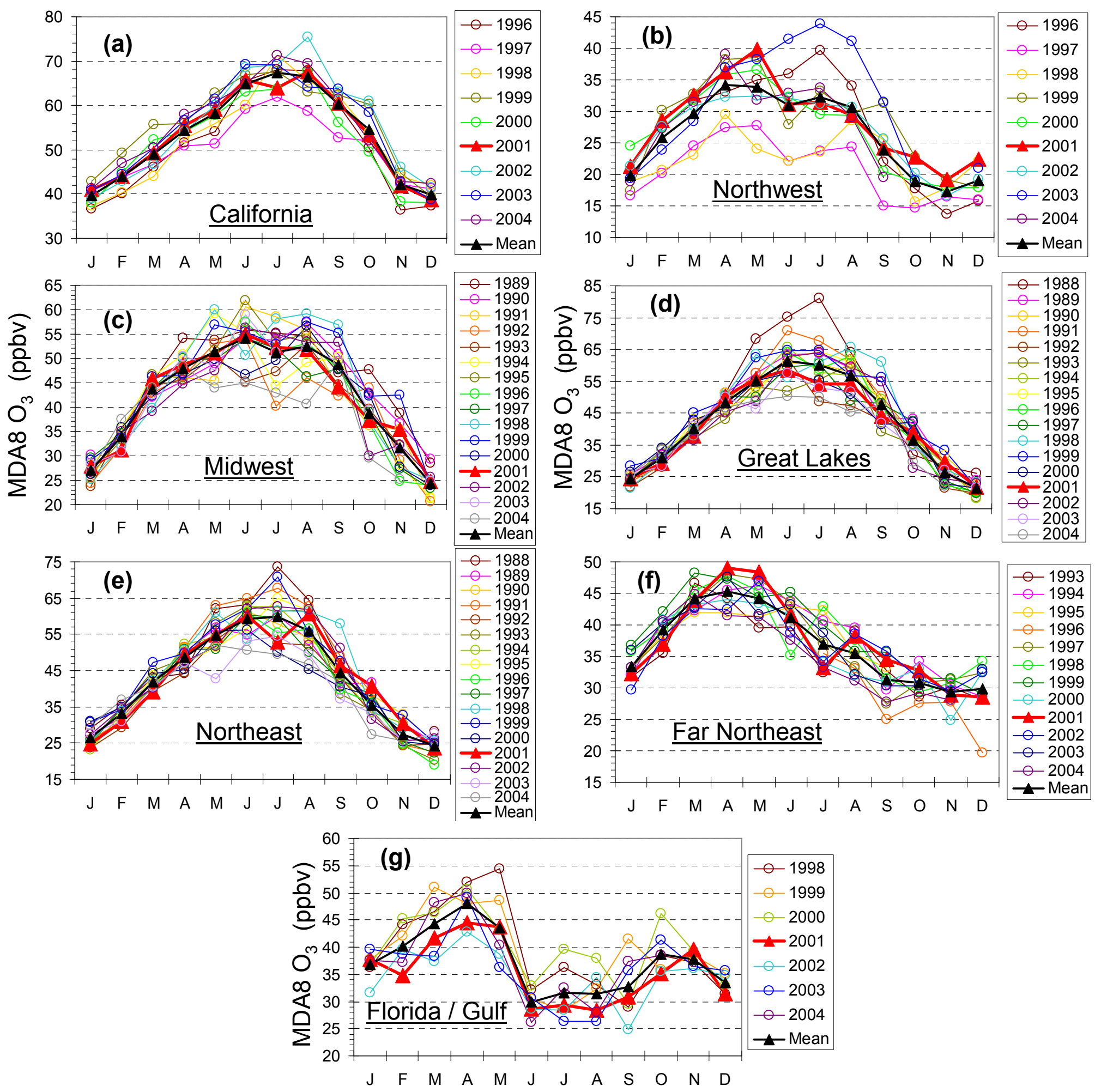

Figure A2. As in Fig. 3, but for the other seven regions. 
Table A2. Details of the global models used in our analysis, including emissions inventories and meteorological fields employed for the HTAP simulations. Further description of model characteristics is available at: http:/www.mi.uni-hamburg.de/Listclassification-and-detail-view-of-model-entr.567.0.html. Adapted from Fiore et al. [2009].

\begin{tabular}{|c|c|c|c|c|c|}
\hline Model & $\begin{array}{c}\text { Resolution } \\
\text { (Ion x lat x layers) }\end{array}$ & Institution & Model contact & $\begin{array}{c}\text { Anthropogenic emission } \\
\text { inventory for } \mathrm{O}_{3} \\
\text { precursors }\end{array}$ & $\begin{array}{l}\text { Driving } \\
\text { meteorology } \\
\text { (year 2001) }\end{array}$ \\
\hline $\begin{array}{l}\text { CAMCHEM- } \\
3311 \mathrm{~m} 13^{\#}\end{array}$ & $2.5^{\circ} \times 2^{\circ} \times 30$ & NCAR, USA & Peter Hess & $\begin{array}{l}\text { POET for } 1997^{\mathrm{a}} \text {; CO fossil } \\
\text { fuel and biofuel from a } \\
\text { MOPITT inversion }\end{array}$ & NCEP \\
\hline $\begin{array}{l}\text { ECHAM5- } \\
\text { HAMMOZ-v21 }\end{array}$ & $2.81^{\circ} \times 2.81^{\circ} \times 21$ & $\begin{array}{l}\text { EPFL, } \\
\text { Switzerland }\end{array}$ & Gerd Folberth & RETRO & $\begin{array}{l}\text { ECMWF ERA- } \\
40\end{array}$ \\
\hline $\begin{array}{l}\text { EMEP-rv26 }^{\#} \\
\text { (NH only) }\end{array}$ & $1^{\circ} \times 1^{\circ} \times 20$ & EMEP, Norway & $\begin{array}{l}\text { Jan Eiof Jonson, } \\
\text { Peter Wind }\end{array}$ & $\begin{array}{l}\text { IER / Uni-Stuttgart, based } \\
\text { on EDGAR2000; EMEP }\end{array}$ & $\begin{array}{l}\text { ECMWF ERA- } \\
40\end{array}$ \\
\hline $\begin{array}{c}\text { FRSGC/UCI } \\
\mathrm{v} 01^{\#} \\
\end{array}$ & $2.81^{\circ} \times 2.81^{\circ} \times 37$ & $\begin{array}{c}\text { Lancaster } \\
\text { University, UK } \\
\end{array}$ & Oliver Wild & ACCENT/AR4 ${ }^{c}$ & ECMWF IFS $^{d}$ \\
\hline GEMAQ-EC & $2^{\circ} \times 2^{\circ} \times 20$ & $\begin{array}{l}\text { Environment } \\
\text { Canada }\end{array}$ & Sunling Gong & $\begin{array}{c}\text { AURAMS (regional } \\
\text { Canadian, US and Mexico); } \\
\text { EDGAR elsewhere }\end{array}$ & $\begin{array}{c}\text { Canadian } \\
\text { Meteorological } \\
\text { Centre (CMC) }\end{array}$ \\
\hline GEMAQ-v1po ${ }^{\#}$ & $4^{\circ} \times 4^{\circ} \times 28$ & $\begin{array}{c}\text { York } \\
\text { University, } \\
\text { Canada } \\
\end{array}$ & Alexandru Lupu & EDGAR v2 & CMC \\
\hline $\begin{array}{c}\text { GEOS-Chem } \\
\text { v07 }\end{array}$ & $2.5^{\circ} \times 2^{\circ} \times 30$ & $\begin{array}{c}\text { Harvard } \\
\text { University, } \\
\text { USA } \\
\end{array}$ & Rokjin Park & Bey et al., $2001^{\mathrm{e}}$ & NASA GEOS-4 \\
\hline $\begin{array}{l}\text { GEOS-Chem } \\
\text { v07-res } 4 \times 5\end{array}$ & $5^{\circ} \times 4^{\circ} \times 30$ & CIEMAT, Spain & $\begin{array}{l}\text { Marta Garcia } \\
\text { Vivanco } \\
\end{array}$ & $\begin{array}{c}\text { EMEP emissions } \\
\text { EPA/NEI99 inventory }\end{array}$ & NASA GEOS-4 \\
\hline $\begin{array}{l}\text { GISS-PUCCNI- } \\
\text { modelE }^{\#}\end{array}$ & $5^{\circ} \times 4^{\circ} \times 23$ & $\begin{array}{c}\text { NASA GISS, } \\
\text { USA }\end{array}$ & Drew Shindell & $\begin{array}{c}\text { ACCENT / AR4, with EA } \\
\text { emissions of } \mathrm{CO} \text { and } \mathrm{NO}_{\mathrm{x}} \\
\text { times } 1.66\end{array}$ & $\begin{array}{l}\text { NCEP, via linear } \\
\text { relaxation }\end{array}$ \\
\hline GMI-v02f $f^{\#}$ & $2.5^{\circ} \times 2^{\circ} \times 42$ & $\begin{array}{l}\text { NASA GSFC, } \\
\text { USA }\end{array}$ & Bryan Duncan & $\begin{array}{c}\text { Harvard's merged inventory } \\
\text { (NEI99, BRAVO, Streets, } \\
\text { EMEP) }\end{array}$ & NASA GEOS-4 \\
\hline LMDz3-INCA1 ${ }^{\#}$ & $3.75^{\circ} \times 2.5^{\circ} \times 19$ & LSCE, France & Sophie Szopa & RETRO & $\begin{array}{l}\text { ECMWF ERA- } \\
40 \\
\end{array}$ \\
\hline $\begin{array}{c}\text { LLNL-IMPACT- } \\
\text { T5a }^{\#}\end{array}$ & $2.5^{\circ} \times 2^{\circ} \times 48$ & LLNL, USA & $\begin{array}{l}\text { Cynthia Atherton, } \\
\text { Daniel Bergmann }\end{array}$ & POET & $\begin{array}{l}\text { NASA GEOS-4- } \\
\text { ceres }\end{array}$ \\
\hline $\begin{array}{l}\text { MOZART- } \\
\text { GFDL-v2 }^{\#}\end{array}$ & $1.88^{\circ} \times 1.88^{\circ} \times 28$ & GFDL, USA & Arlene Fiore & EDGAR v2 & NCEP \\
\hline MOZECH-v16 & $2.81 \times 2.81 \times 31$ & $\begin{array}{l}\text { FZ Jülich, } \\
\text { Germany }\end{array}$ & $\begin{array}{l}\text { Martin Schultz, } \\
\text { Sabine Schröder }\end{array}$ & RETRO & $\begin{array}{l}\text { ECMWF ERA- } \\
\qquad 40\end{array}$ \\
\hline Oslo CTM2 & $2.81^{\circ} \times 2.81^{\circ} \times 40$ & $\begin{array}{l}\text { University of } \\
\text { Oslo, Norway }\end{array}$ & Michael Gauss & EDGAR v3.2 & ECMWF - IFS \\
\hline $\begin{array}{l}\text { TM5-JRC-cy2- } \\
\text { ipcc-v1 }{ }^{\#}\end{array}$ & $1^{\circ} \times 1^{\circ} \times 25$ & JRC, Italy & $\begin{array}{l}\text { Frank Dentener, } \\
\text { Elina Marmer }\end{array}$ & ACCENT / AR4 & ECMWF \\
\hline
\end{tabular}

\# - Model used in $\mathrm{NO}_{\mathrm{x}}+\mathrm{CO}+\mathrm{VOC}+$ aerosol emission perturbation simulations (all 16 used for "base-case" comparisons)

a - Granier, C. et al. (2004), Present and future surface emissions of atmospheric compounds, European

Commission report EVK 2199900011. (Available at http:// http://www.aero.jussieu.fr/projet/ACCENT/POET.php)

b - Pétron, G., C. Granier, B. Khattatov, V. Yudin, J. Lamarque, L. Emmons, J. Gille, and D. P. Edwards

(2004), Monthly CO surface sources inventory based on the 2000 - 2001 MOPITT satellite data, Geophys.

Res. Lett., 31, L21107, doi:10.1029/2004GL020560.

c - Bond, T.C. et al. (2004), A technology-based global inventory of black and organic carbon emissions from combustion, J. Geophys. Res., 109, D14203, doi:10.1029/2003JD003697.

d - van der Werf, G.R., J.T. Randerson, L. Giglio, G.J. Collatz, P.S. Kasibhatla, and A.F. Arellano, Jr. (2006), Interannual variability in global biomass burning emissions from1997 to 2004, Atmos. Chem.

Physics, 6, 3423-3441.

e - based on EDGAR v3.2, see Stevenson et al. [2006] 


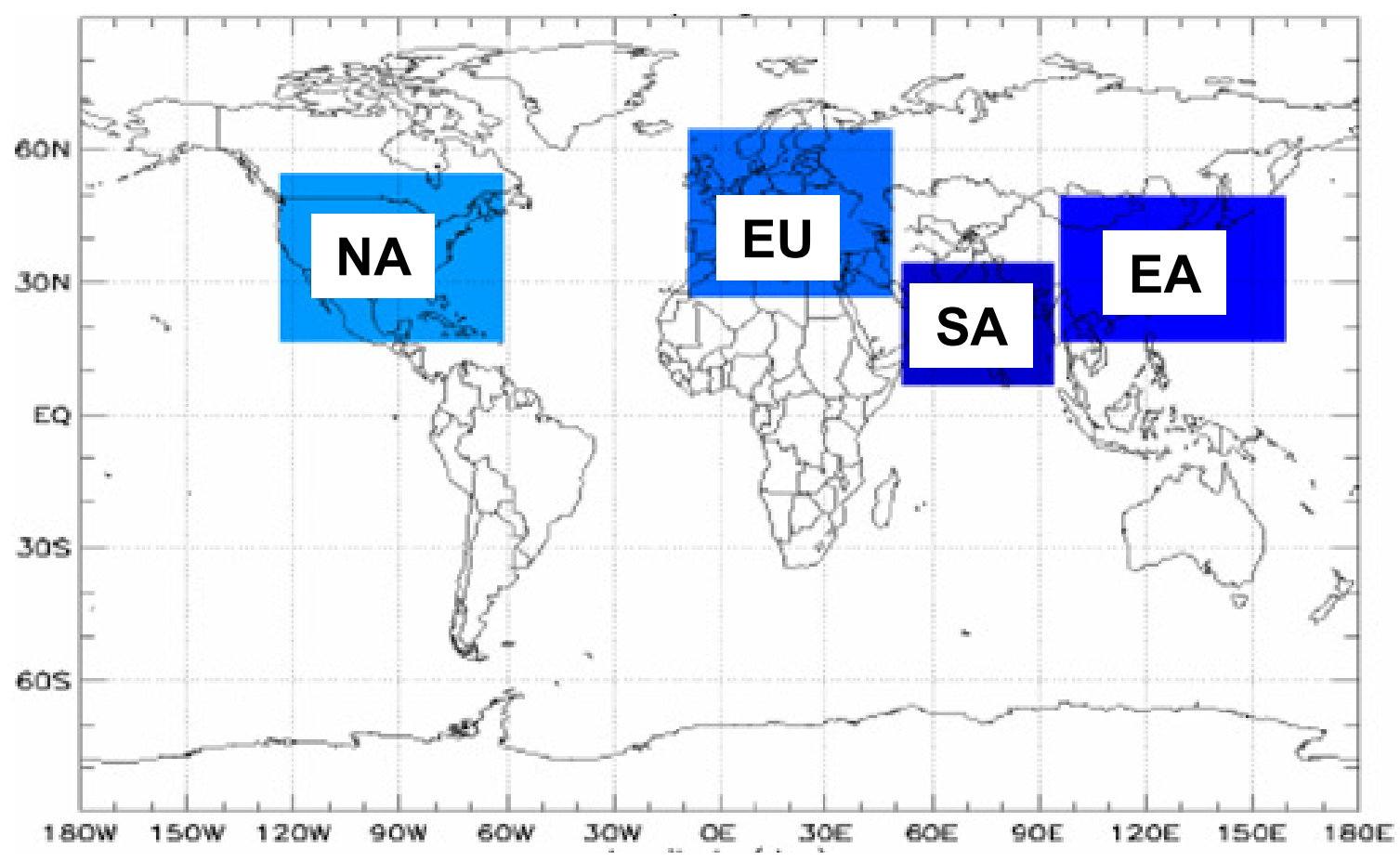

Figure A3. Colored boxes represent the four source regions used in the HTAP emissions reductions simulations: NA - North America, EU - Europe, SA - South Asia, EA - East Asia [adapted from TF HTAP, 2007]. 


\begin{tabular}{|lll|}
\hline$\square$ CAMCHEM & $\square-$ ECHAM5 & $\square$ EMEP \\
$\square-$ FRSGCUCI & $\square$ GEMAQ-EC & $\square$ GEMAQ-v1p0 \\
$\square$ GEOSChem-v07 & $-\square$ GEOSChem-v45 & $\square$ GISS-PUCCINI \\
$\square$ GMI & $-\square$ INCA-vSSz & $\square$ LLNL-IMPACT \\
$\square-$ MOZARTGFDL & $\square$ MOZECH & $\square$ OsloCTM2 \\
$\square-$ TM5-JRC & - OBS & - -Multi-model mean \\
\hline
\end{tabular}
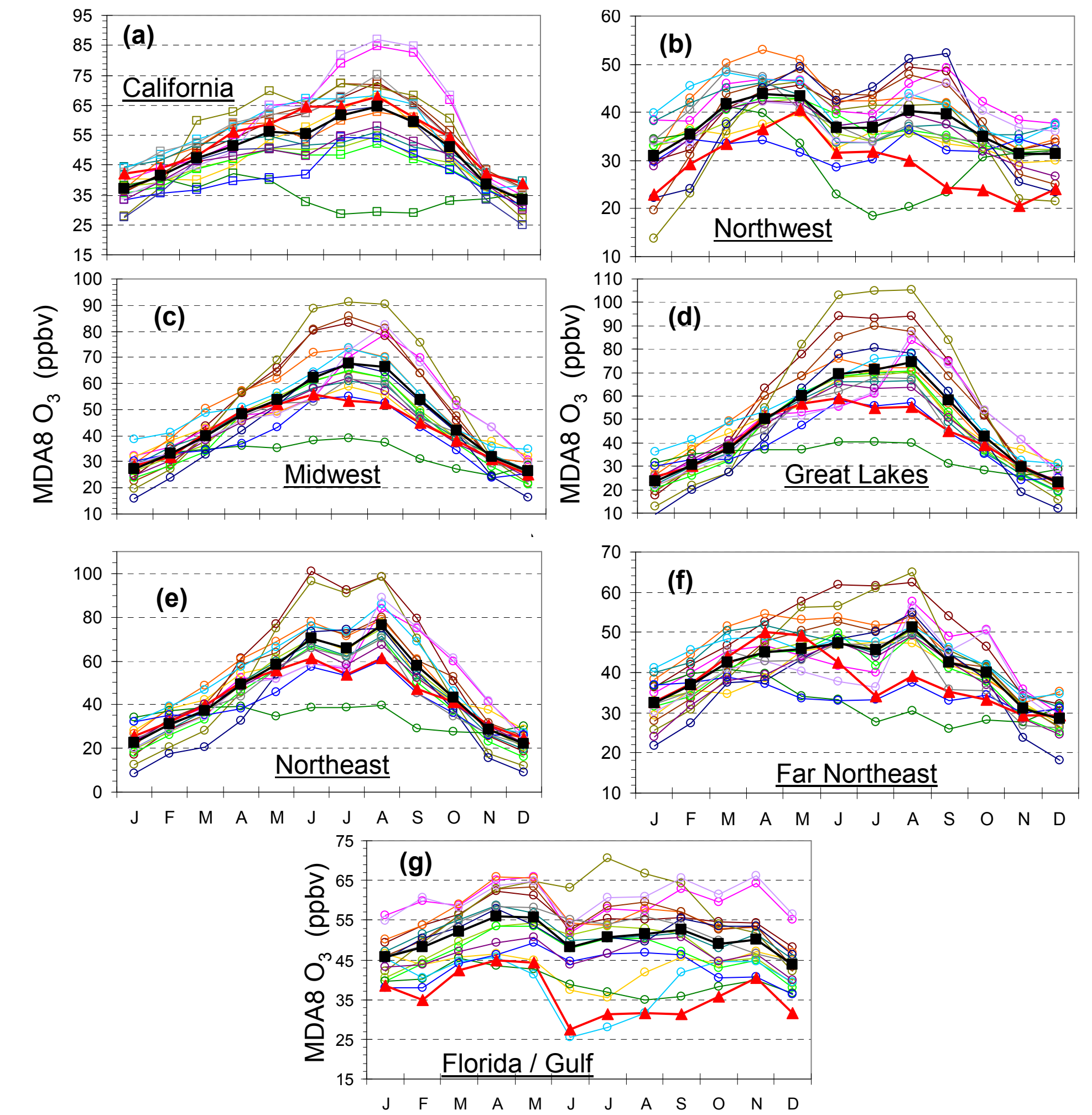

Figure A4. Evaluation of multi-model vs.observed values of $\mathrm{MBDA} \mathrm{O}_{3}$ for the regions not shown in Fig. 4. Individual models are shown with open circles; multi-model mean as solid black squares and observations as solid red triangles. 

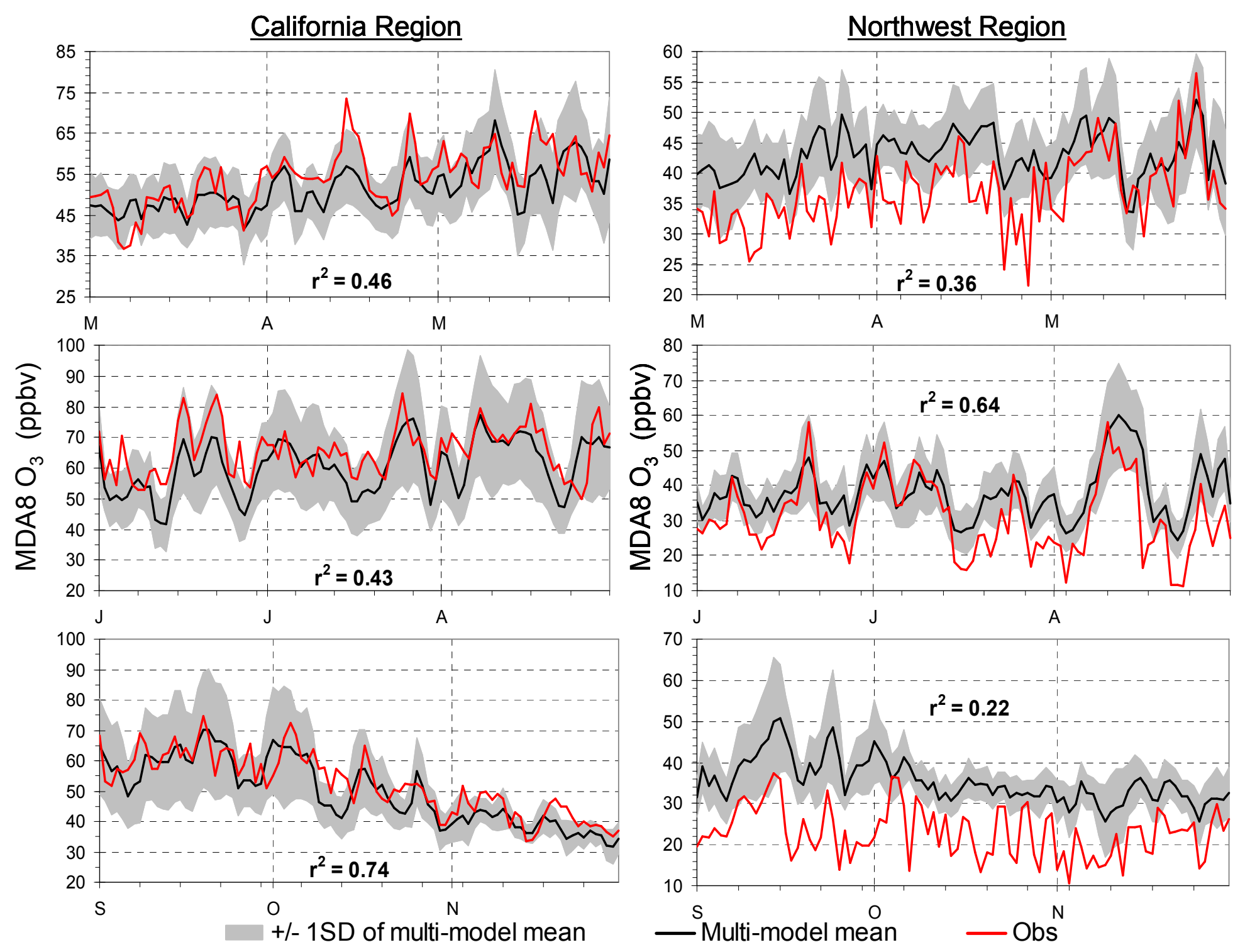

Figure A5. Daily $\mathrm{MDA} 8 \mathrm{O}_{3}$ from observations (red line), multi-model ensemble mean (black line) and $1 \sigma$ of ensemble mean (gray shading) for spring (MAM), summer (JJA) and autumn (SON) in the seven regions not shown in Fig. 5. Note the range of magnitudes on the y-axes. 

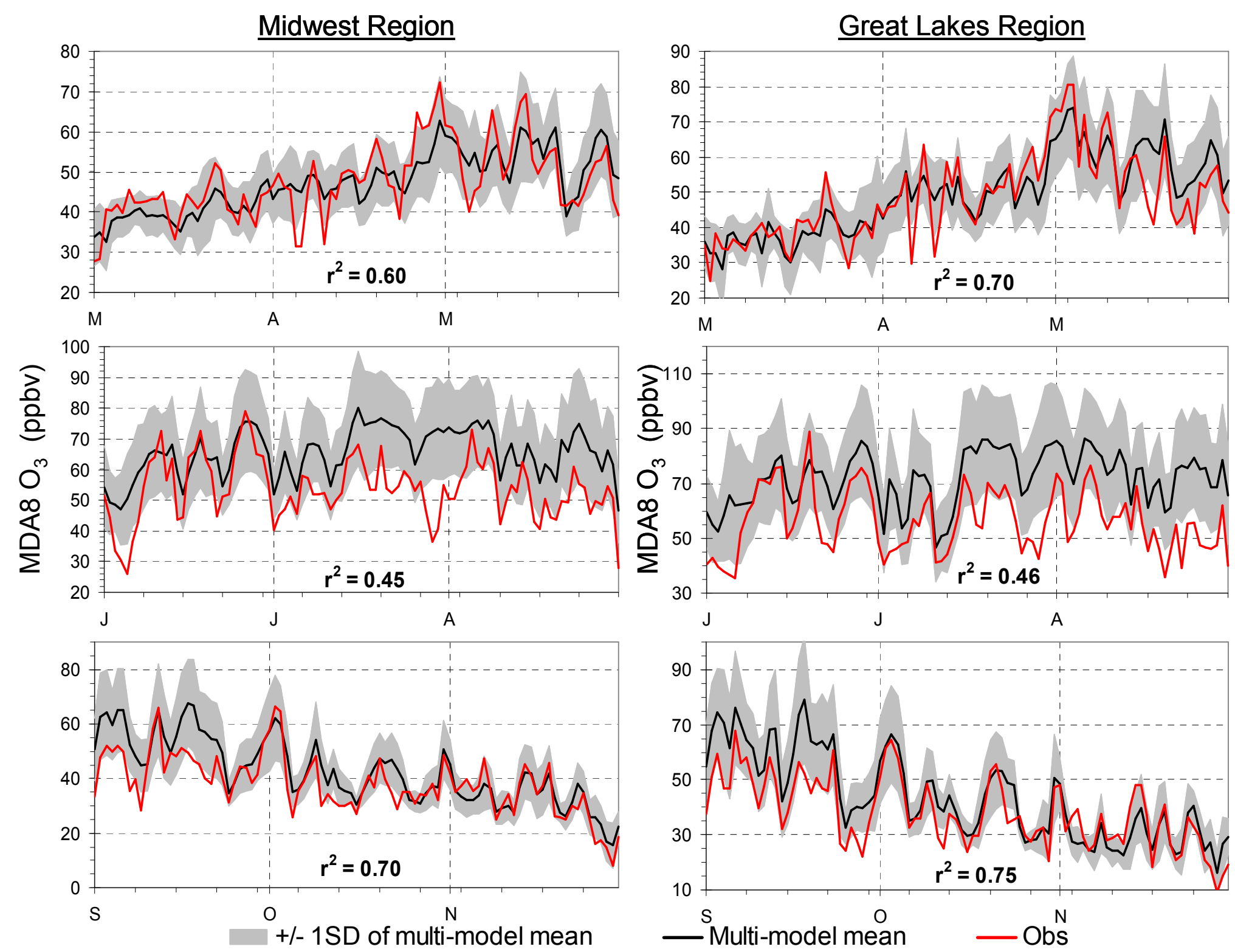

Figure A5. (cont'd) 

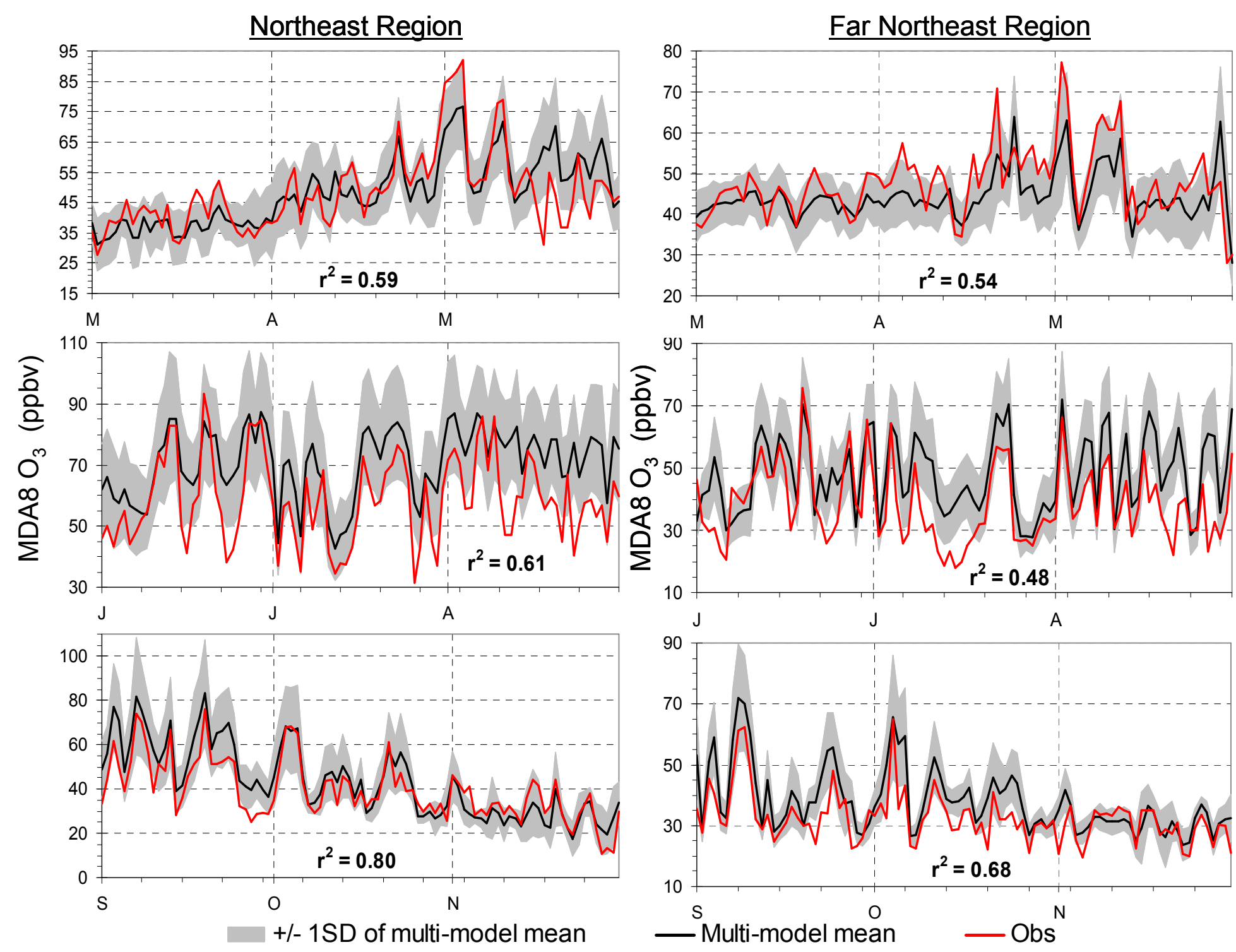

Figure A5. (cont'd) 

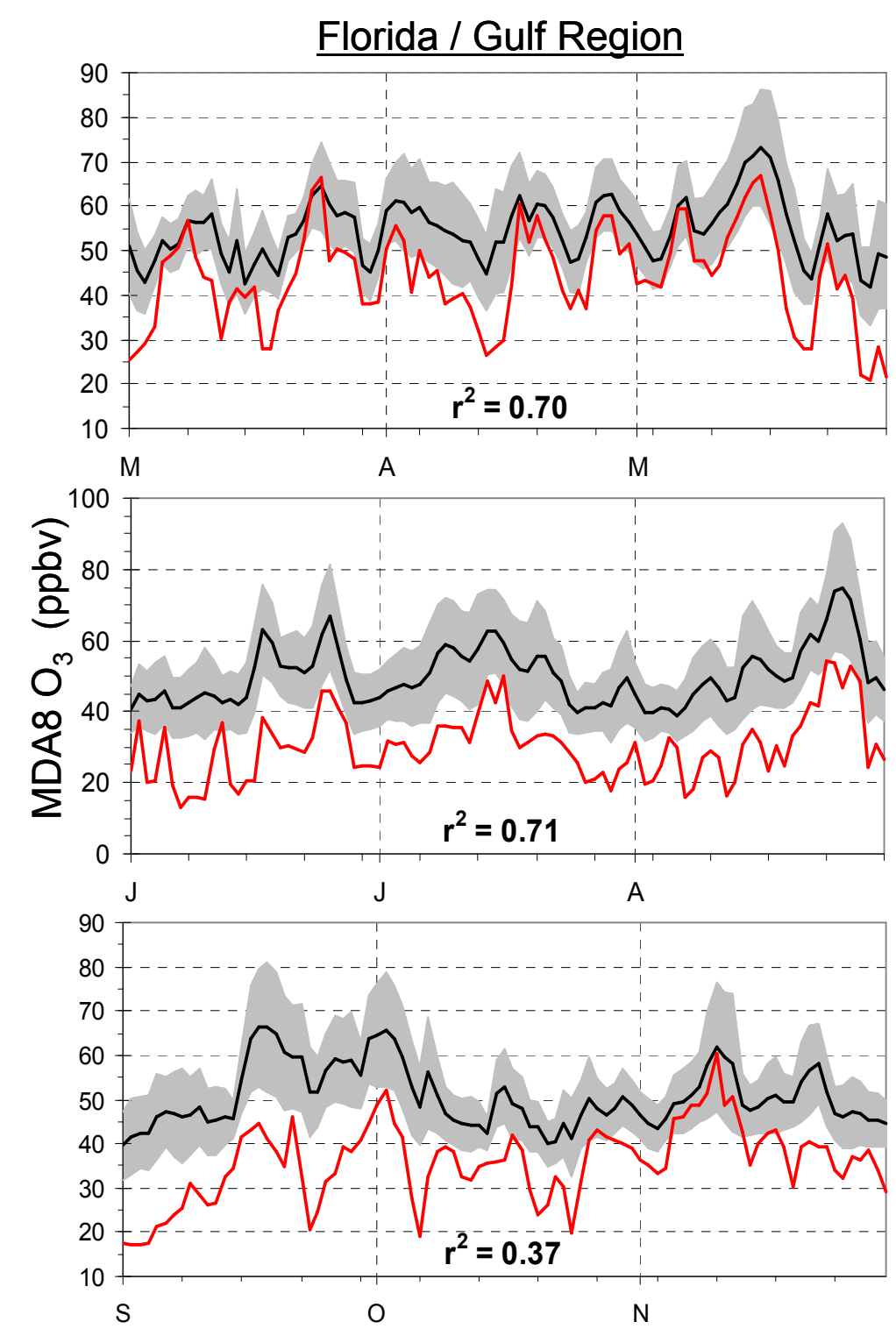

+/- 1SD of multi-model mean _ Multi-model mean

- Obs

Figure A5. (cont'd) 

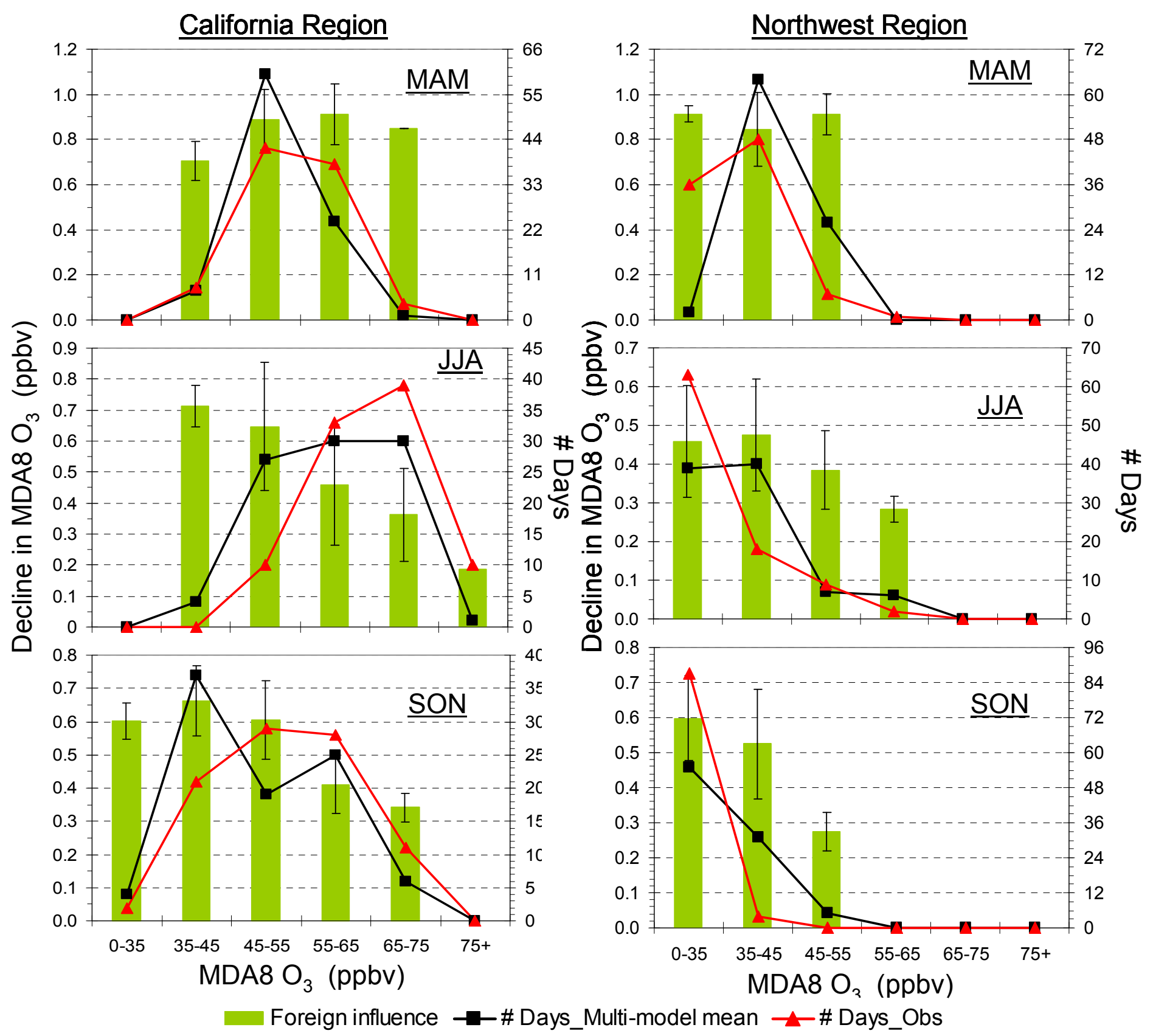

Figure A6. Number of days for each $\mathrm{MDA}_{8} \mathrm{O}_{3}$ bin (right-axis) from the multi-model ensemble (black squares) and observations (red triangles) and the sum of the responses of MDA8 $\mathrm{O}_{3}$ to $20 \%$ reductions in anthropogenic $\mathrm{O}_{3}$-precursor emissions $\left(\mathrm{NO}_{\mathrm{x}}+\mathrm{CO}+\right.$ NMVOC + aerosols) in the three foreign source regions (left-axis; green columns with error bars representing $1 \sigma$ of the multi-model mean) in the seven regions not shown in Fig. 6, binned by simulated MDA8 $\mathrm{O}_{3}$, for spring (MAM), summer (JJA) and autumn $(\mathrm{SON})$. Note the range of magnitudes on the y-axes. 


\section{Midwest Region}
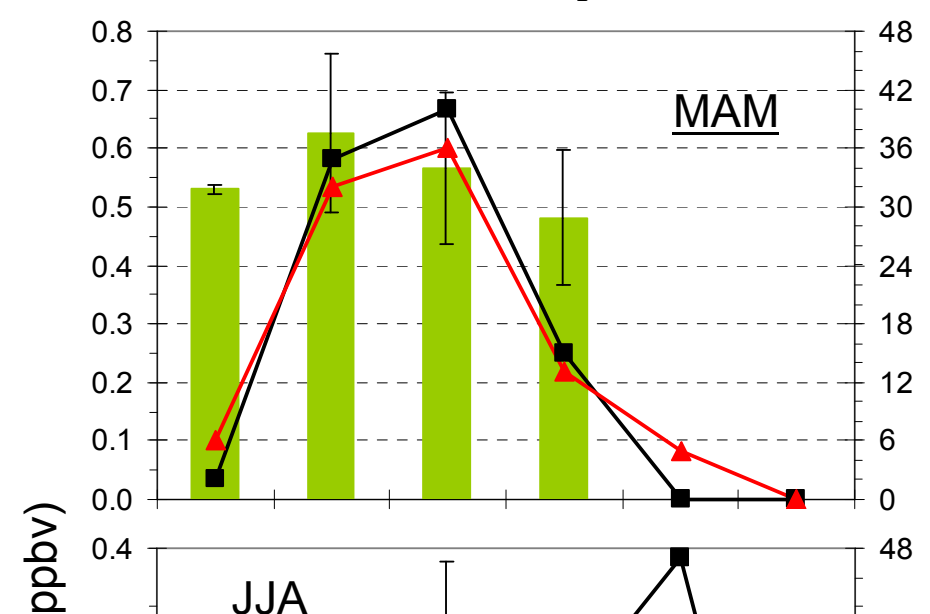

응$$
\text { ด }
$$

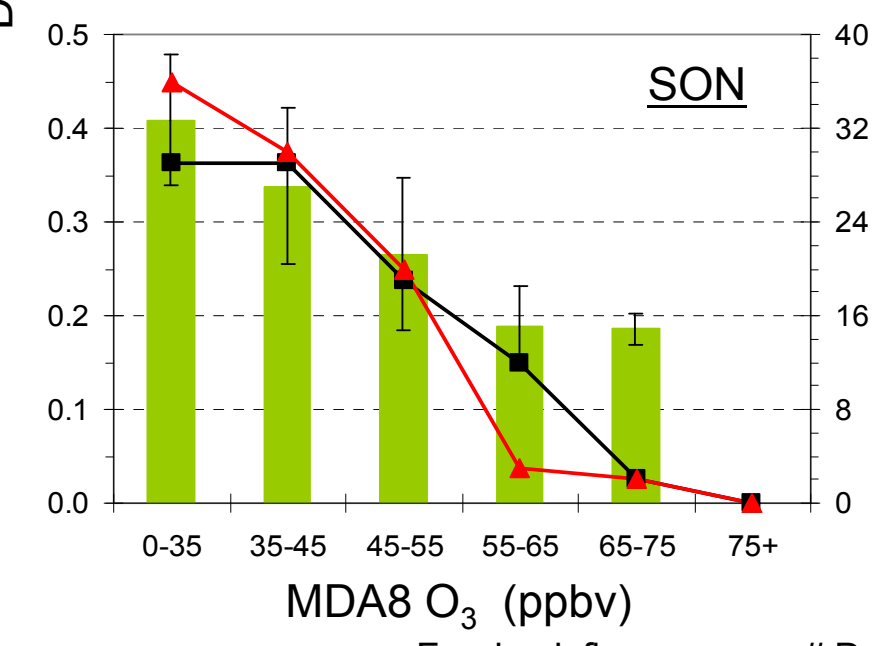

Foreign influence

\section{Great Lakes Region}

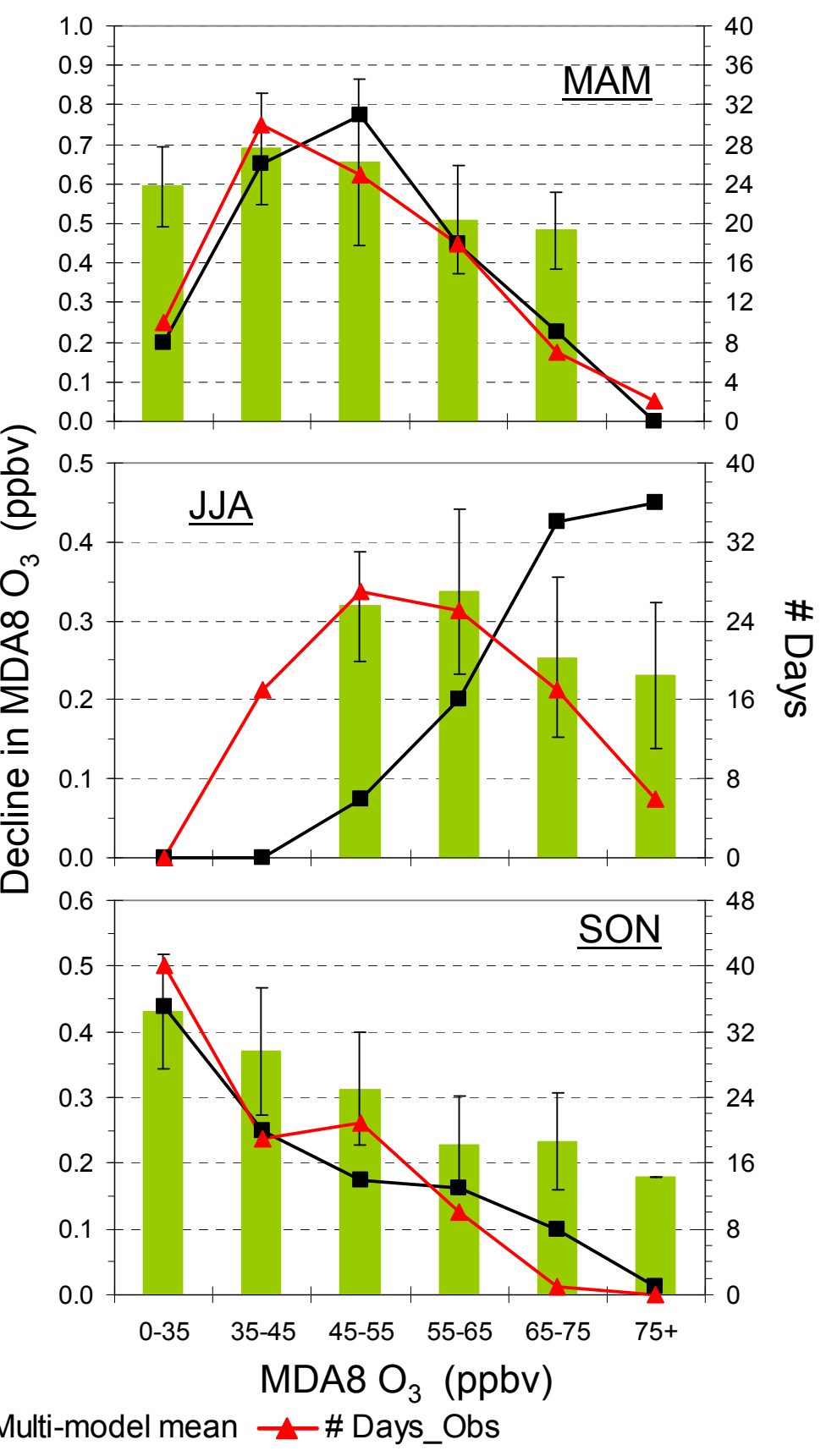

Figure A6. (cont'd) 

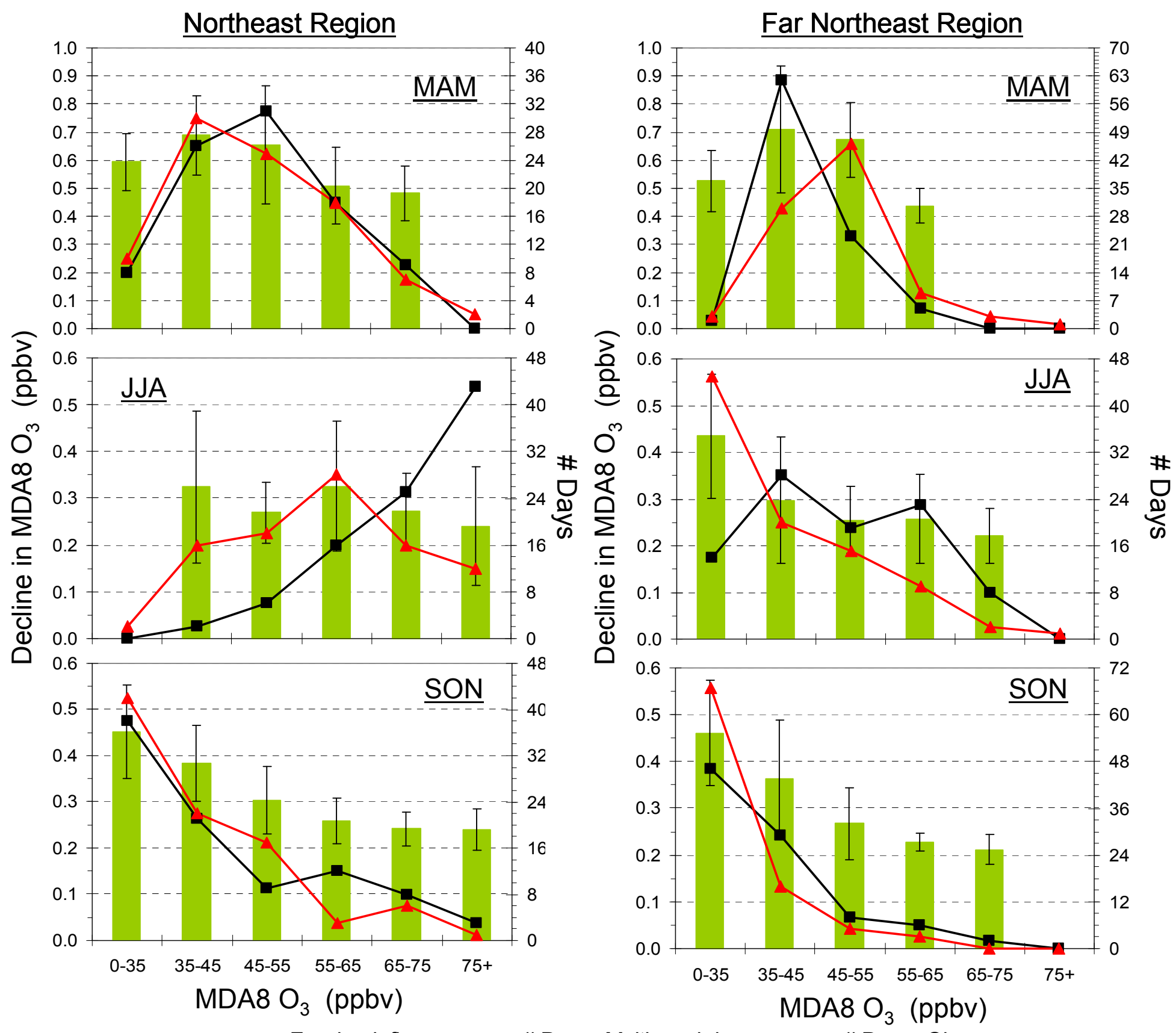

Foreign influence $\rightarrow$-\# Days_Multi-model mean $\rightarrow$-\# Days_Obs

Figure A6. (cont'd) 


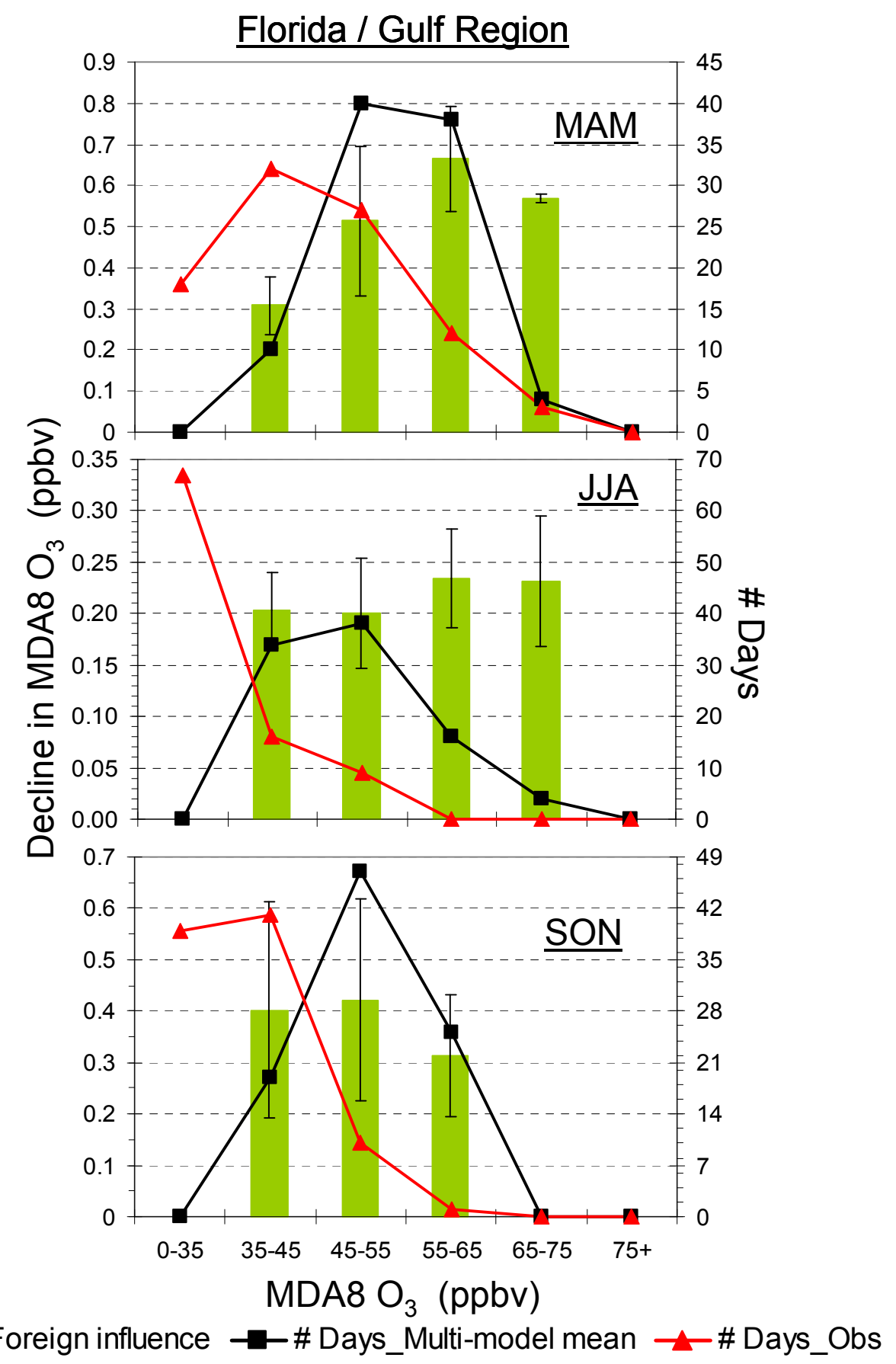

Figure A6. (cont'd) 

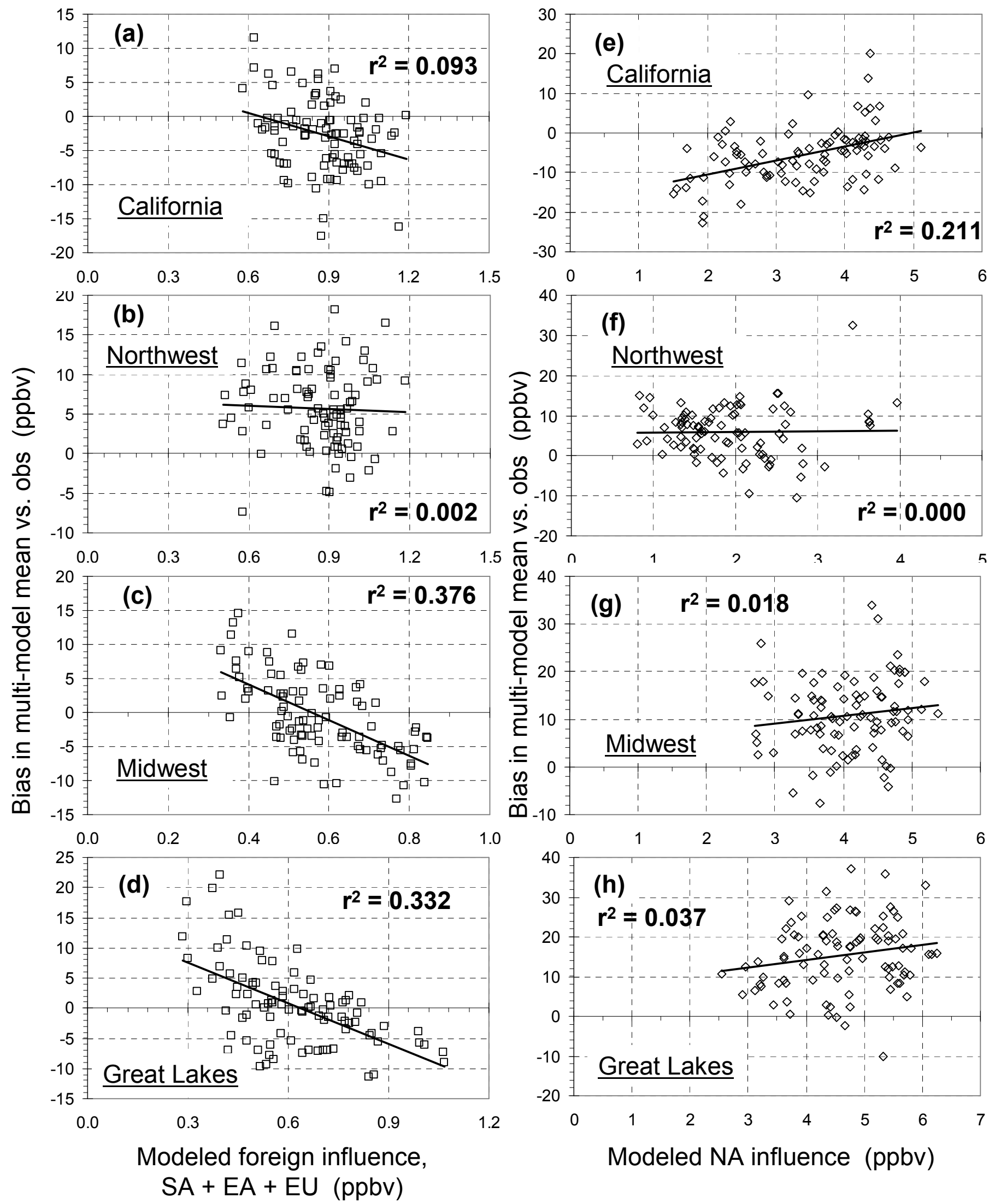

Figure A7. As in Fig. 7, but for the other seven regions. 

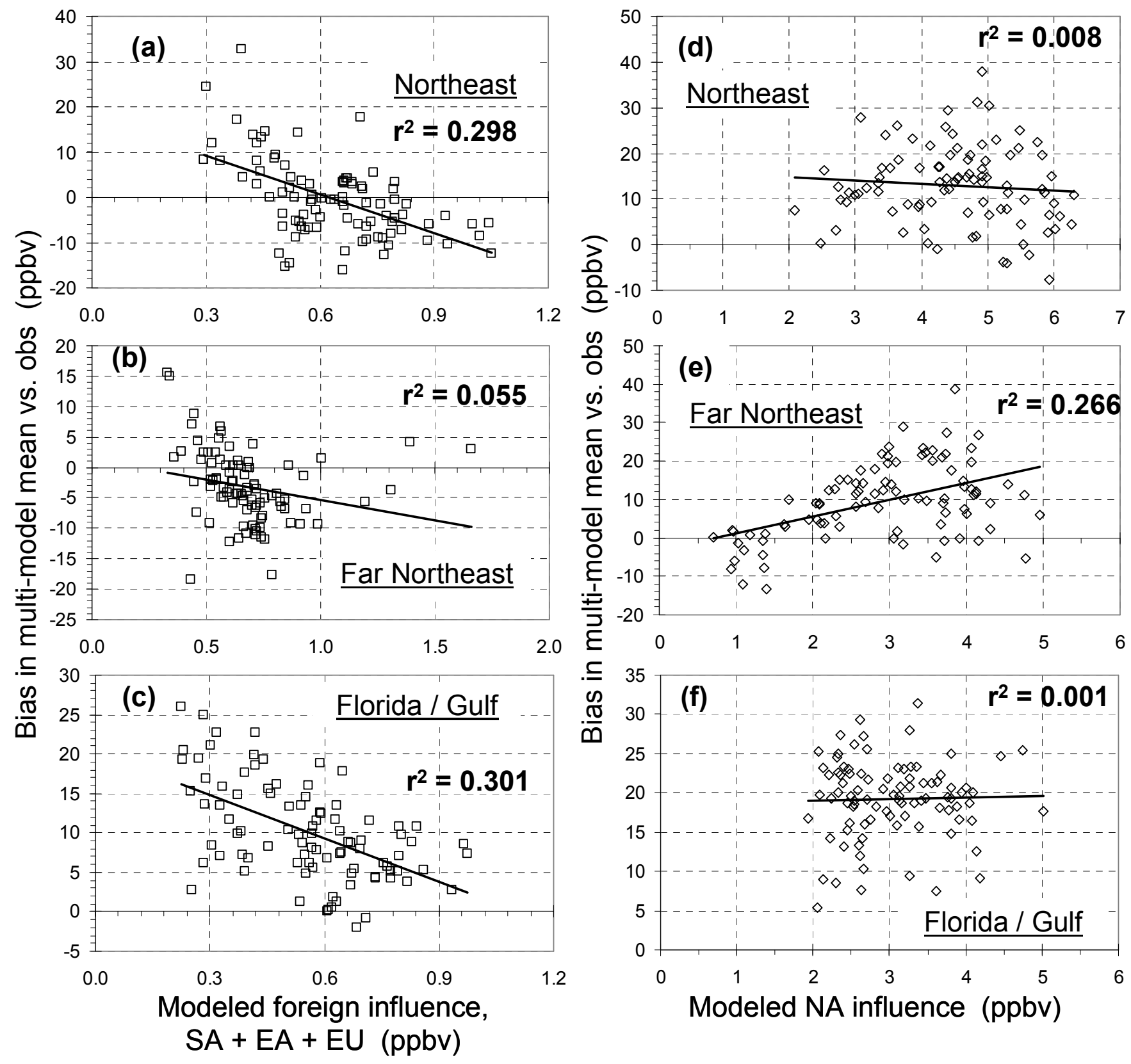

Figure A7. (cont'd) 

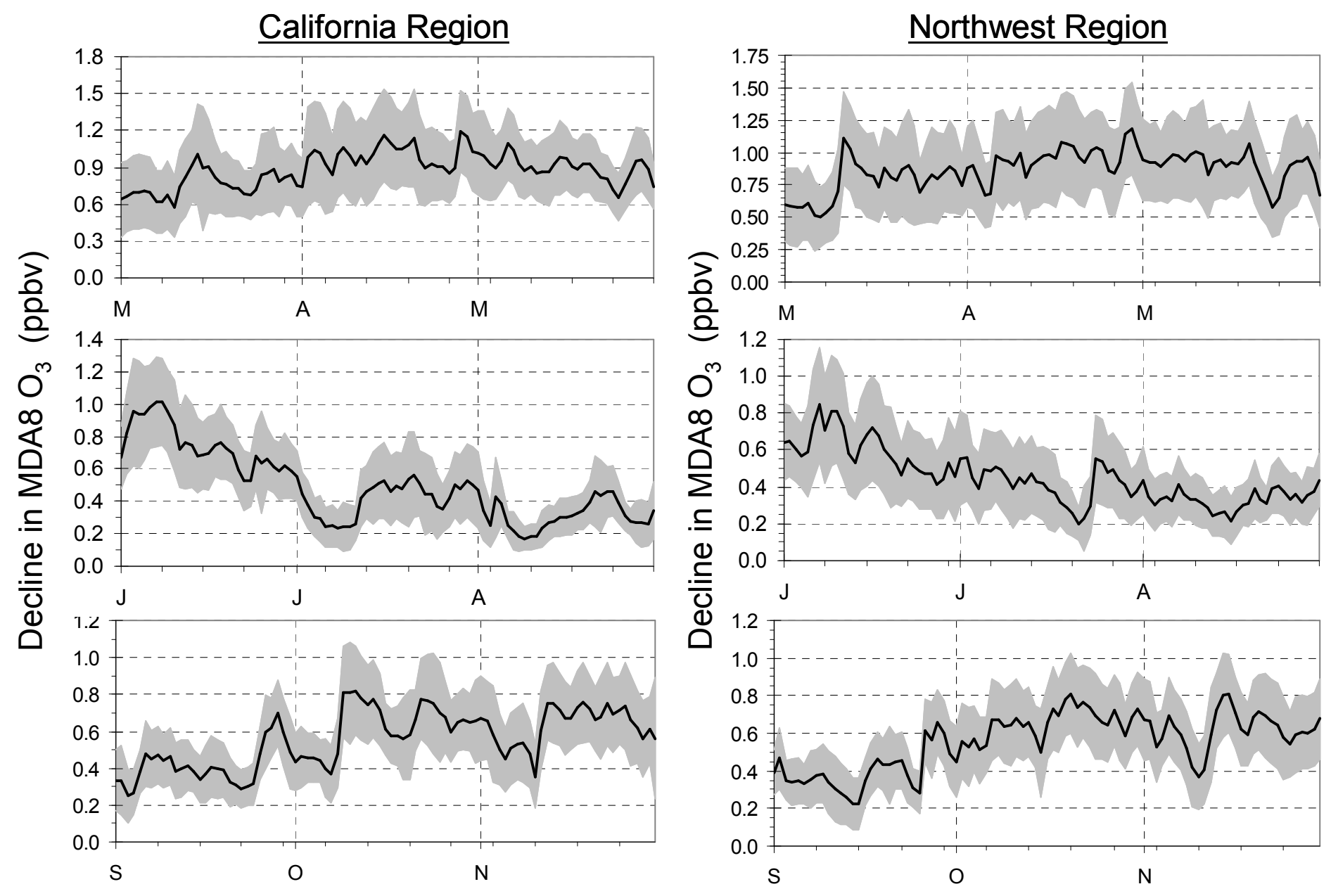

Figure A8. Multi-model mean (black line) and $1 \sigma$ of the ensemble mean (gray shading) in the day-to-day variability of the response of $\mathrm{MDA}_{8} \mathrm{O}_{3}$ to a $20 \%$ reduction in anthropogenic $\mathrm{O}_{3}$-precursor emissions $\left(\mathrm{NO}_{\mathrm{x}}+\mathrm{CO}+\mathrm{NMVOC}+\right.$ aerosols $)$ in the three foreign sources regions (Fig. A3) for the 7 regions not shown in Fig. 8. Note the range of magnitudes on the y-axes. 

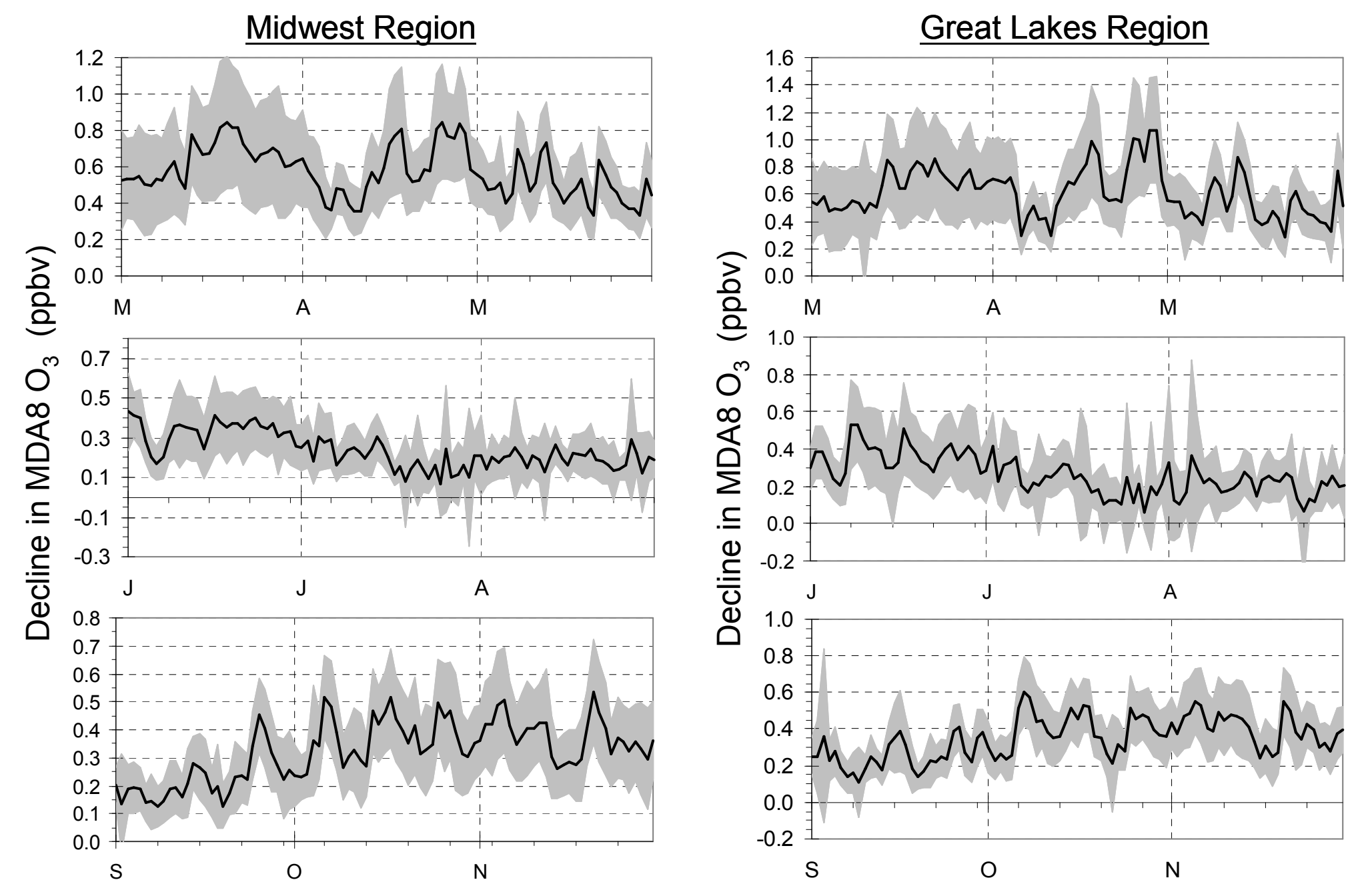

Figure A8. (cont'd) 

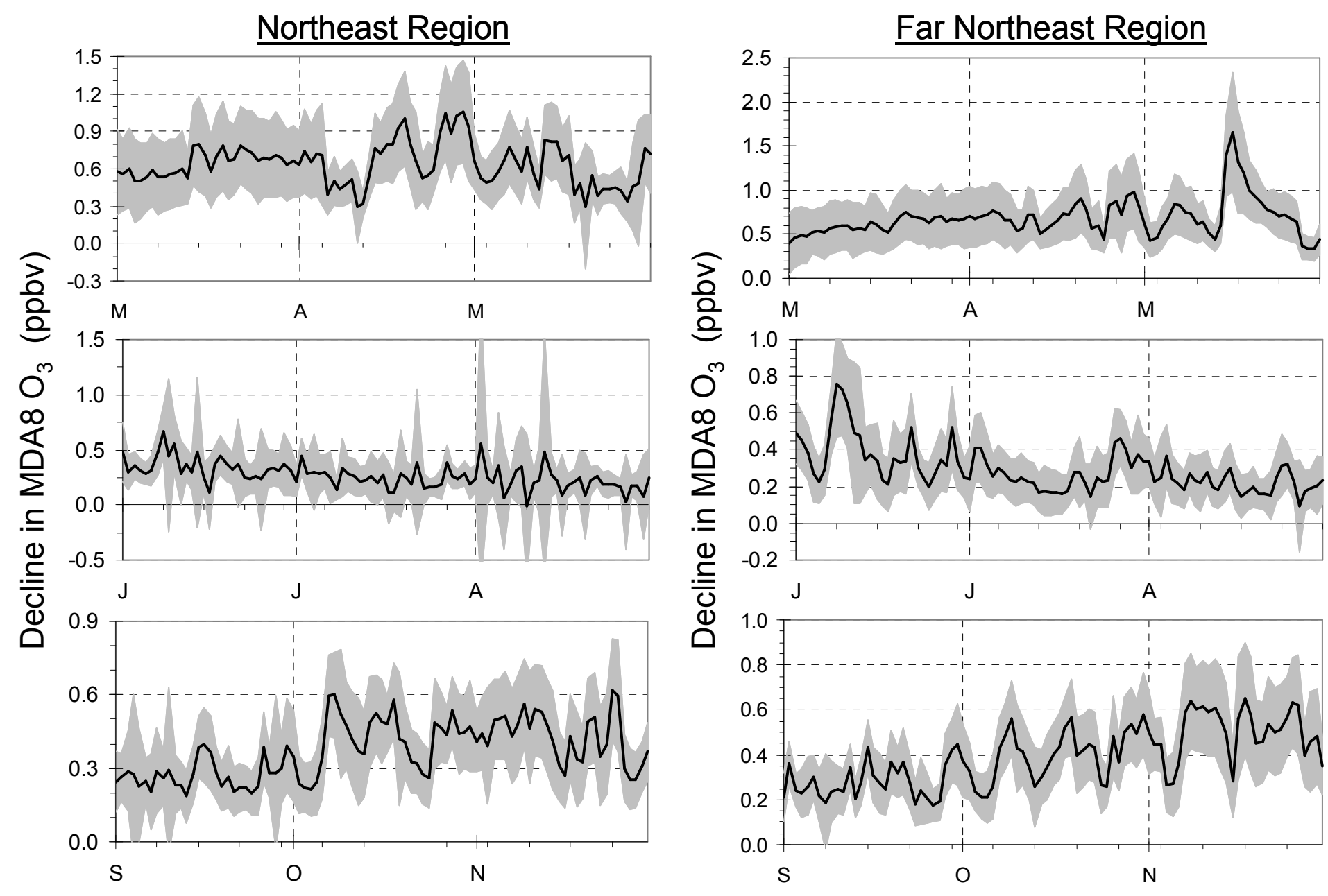

Figure A8. (cont'd) 


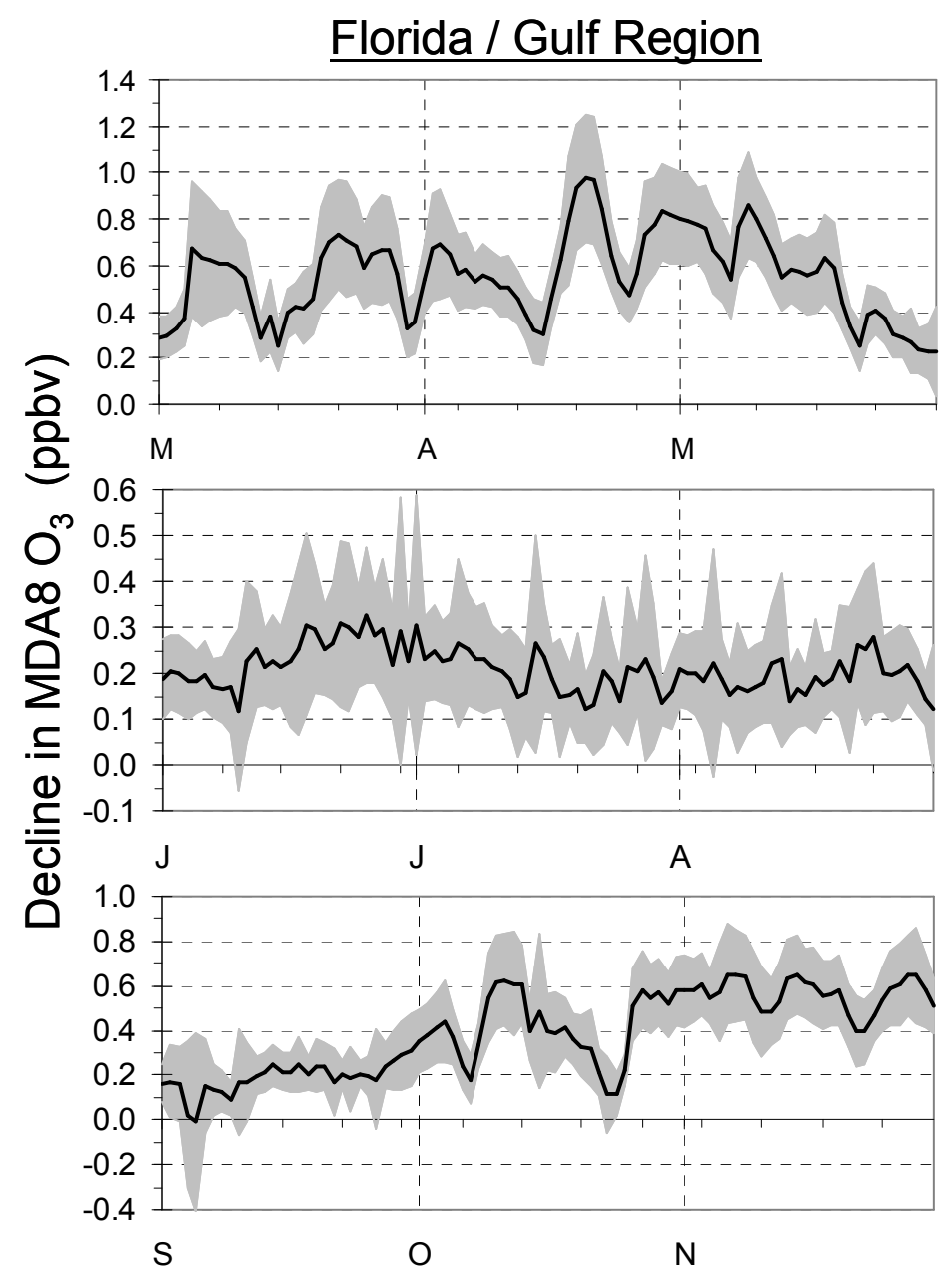

Figure A8. (cont'd) 


\section{California Region}

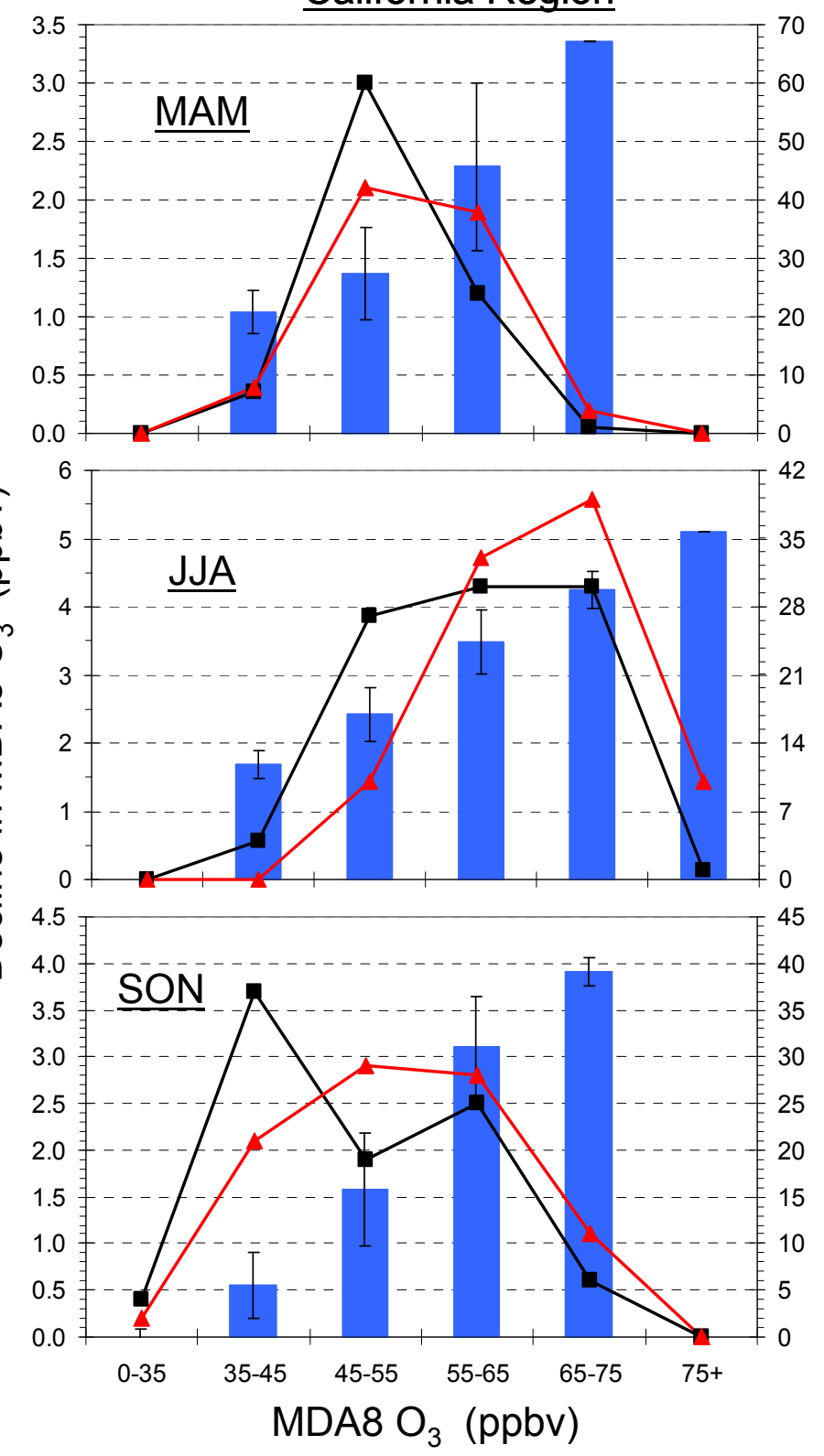

Northwest Region
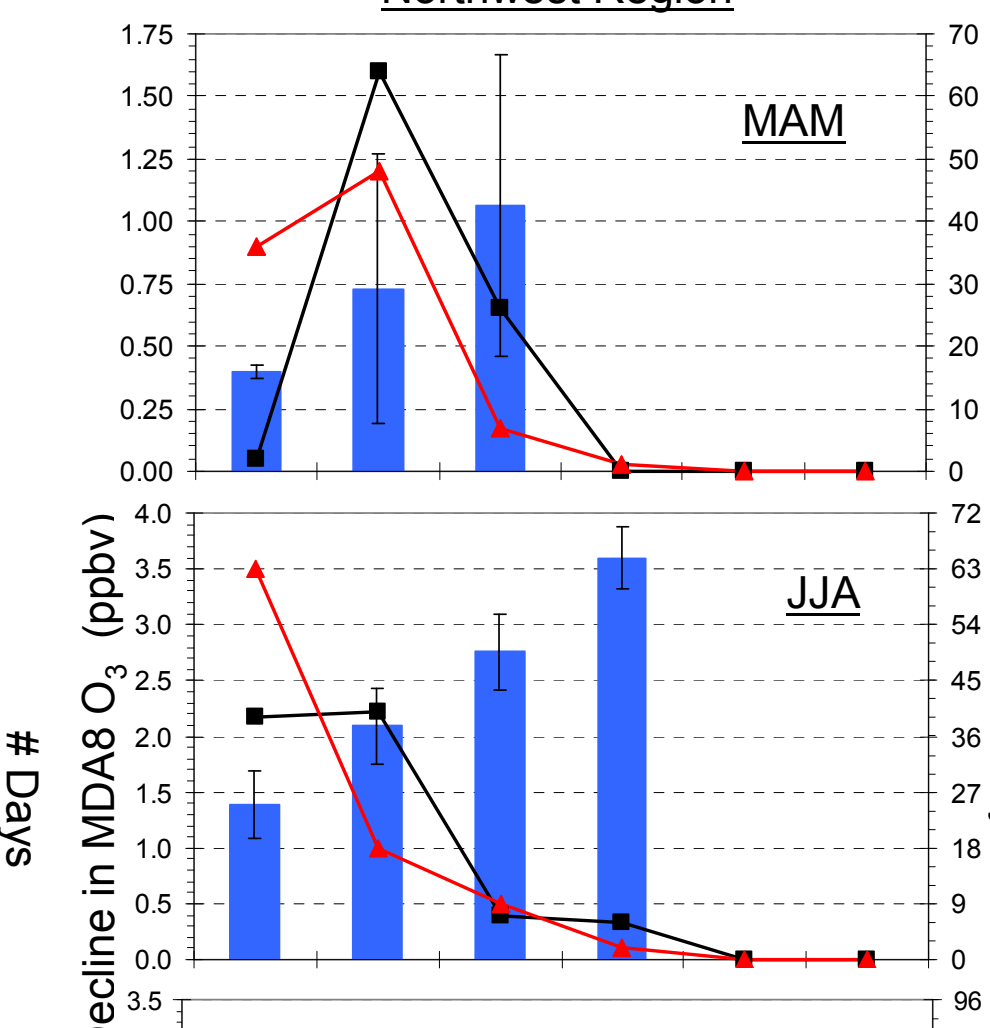

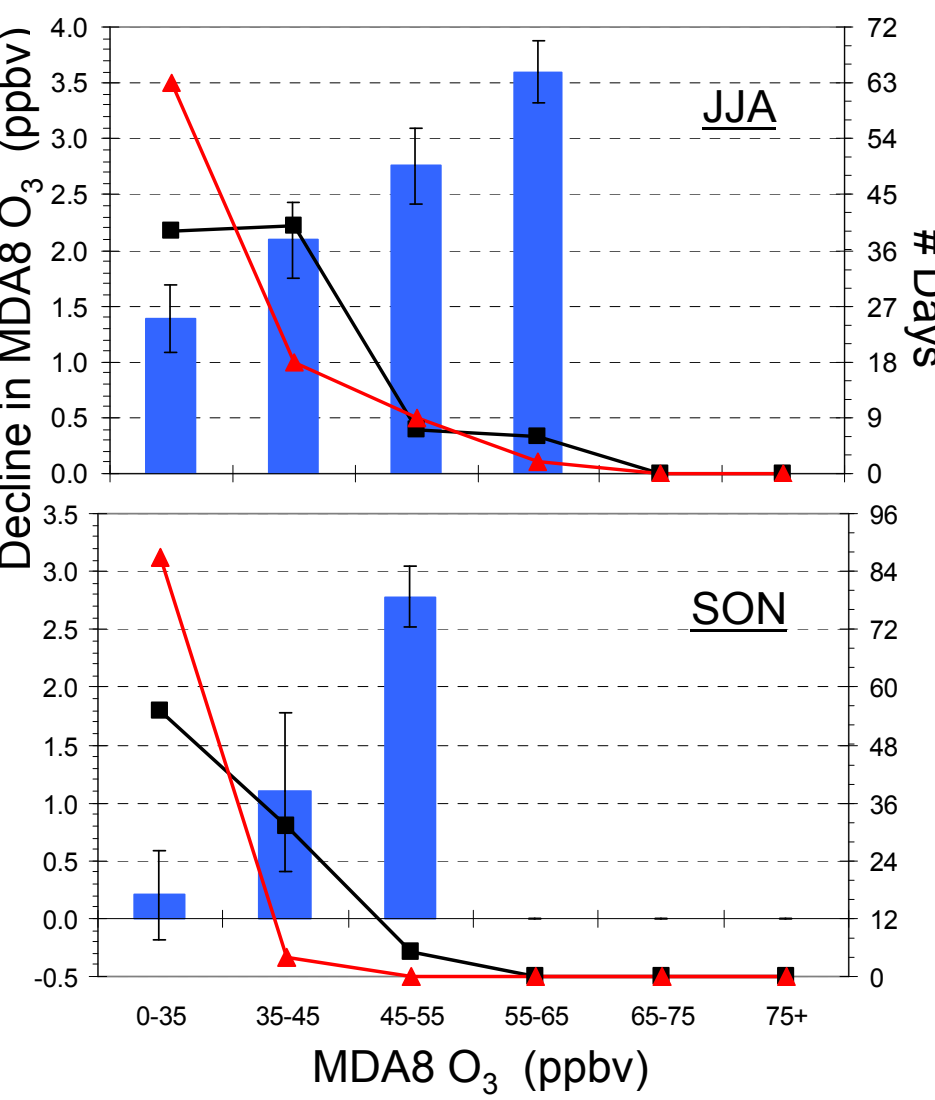

MDA8 $\mathrm{O}_{3}$ (ppbv)

NA influence $\rightarrow-$ \# Days_Multi-model mean $\rightarrow-$ \# Days_Obs

Figure A9. As in Fig. A6, but for a $20 \%$ emissions reduction of anthropogenic $\mathrm{O}_{3}$ precursors $\left(\mathrm{NO}_{\mathrm{x}}+\mathrm{CO}+\mathrm{NMVOC}+\right.$ aerosols $)$ in the North American source region (shown in Fig. A3). Note the range of magnitudes on the y-axes. 

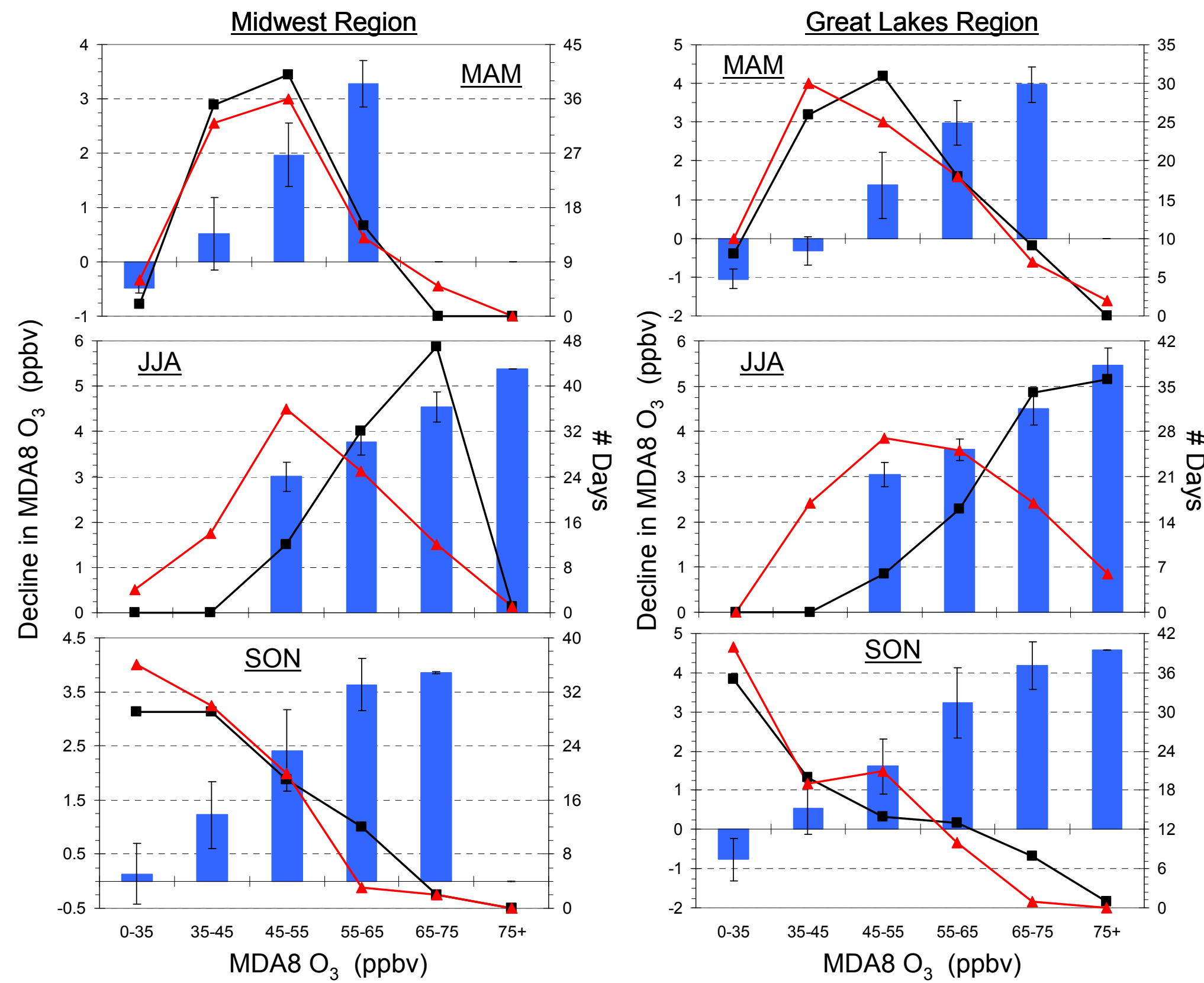

NA influence $\rightarrow$ \# Days_Multi-model mean $\rightarrow$ \# Days_Obs

Figure A9. (cont'd) 

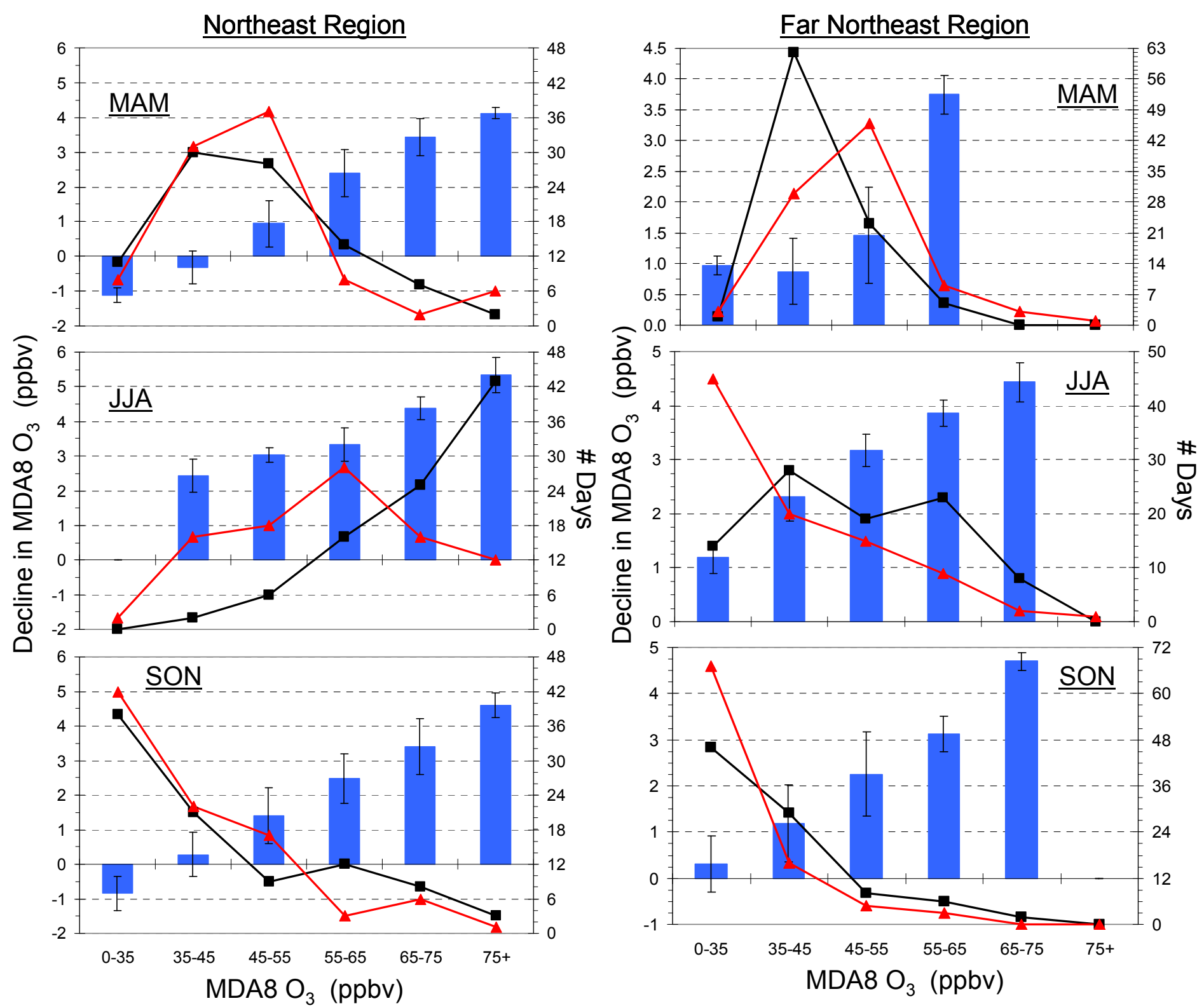

NA influence $\rightarrow-$ \# Days_Multi-model mean $\rightarrow-$ \# Days_Obs

Figure A9. (cont'd) 


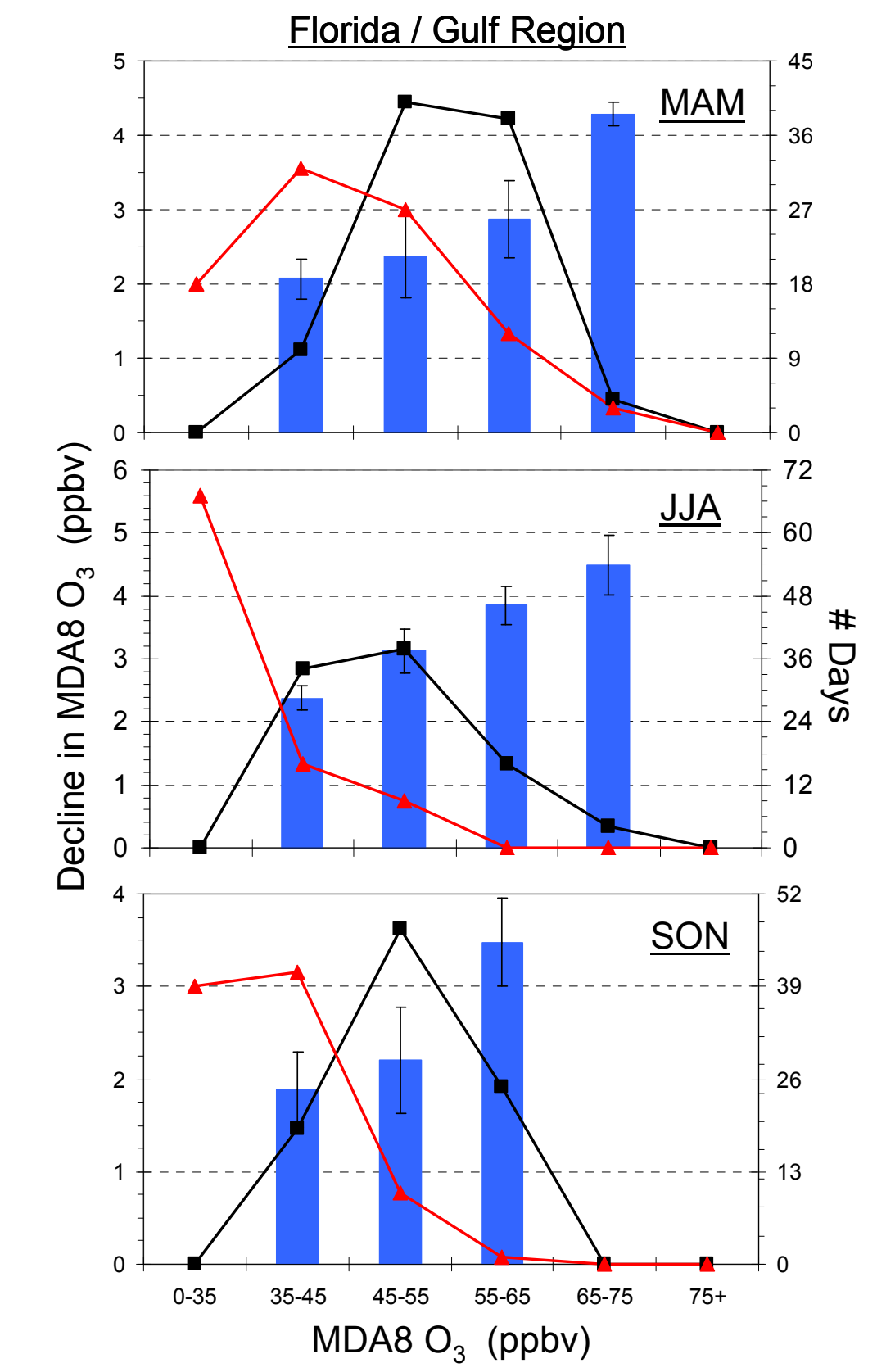

NA influence $\rightarrow-$ \# Days_Multi-model mean $\rightarrow-$ \# Days_Obs

Figure A9. (cont'd) 

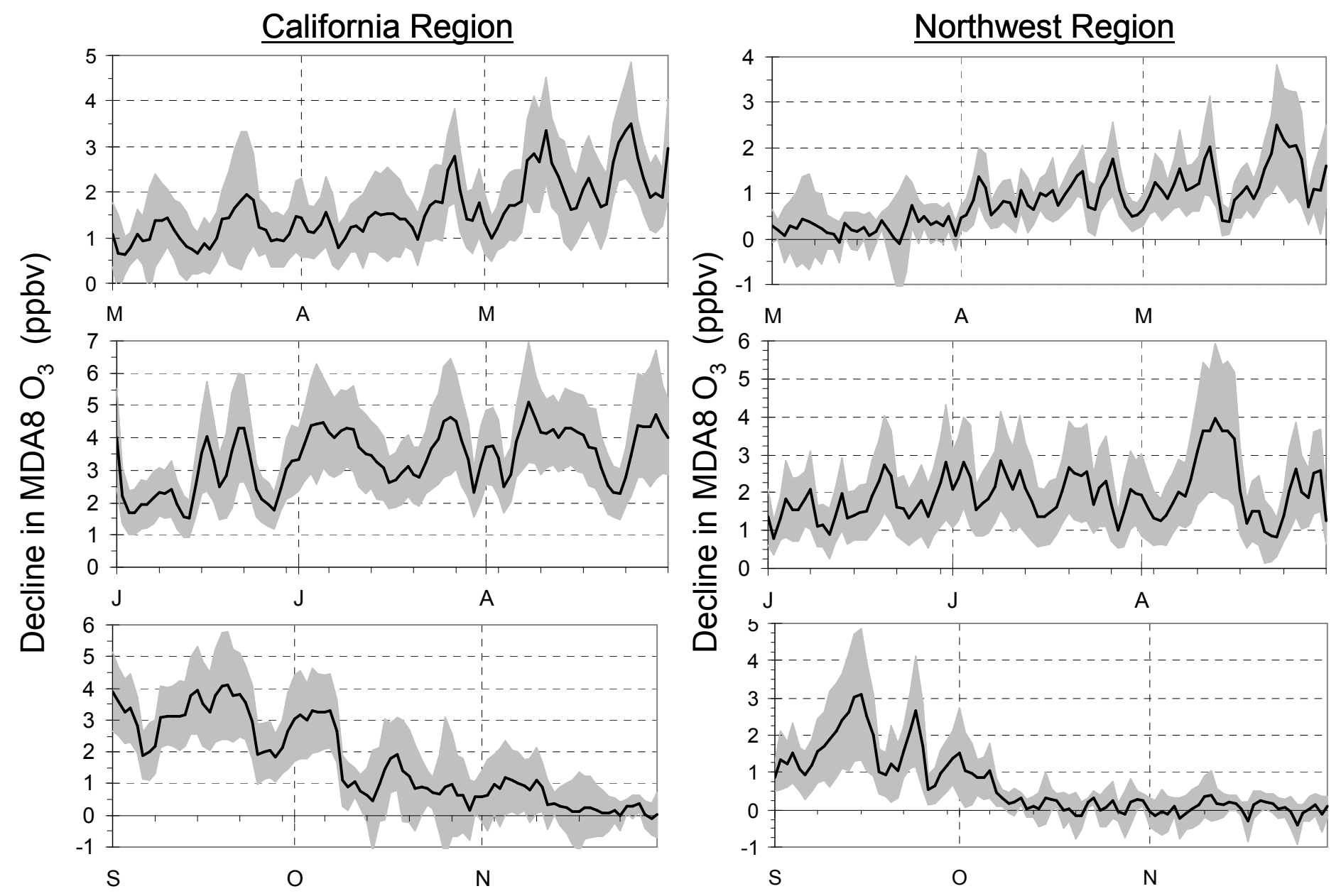

Figure A10. As in Fig. A8, but for a 20\% emissions reduction of anthropogenic $\mathrm{O}_{3}$ precursors $\left(\mathrm{NO}_{\mathrm{x}}+\mathrm{CO}+\mathrm{NMVOC}+\right.$ aerosols $)$ in the North American source region (shown in Fig. A3). Note the range of magnitudes on the y-axes. 

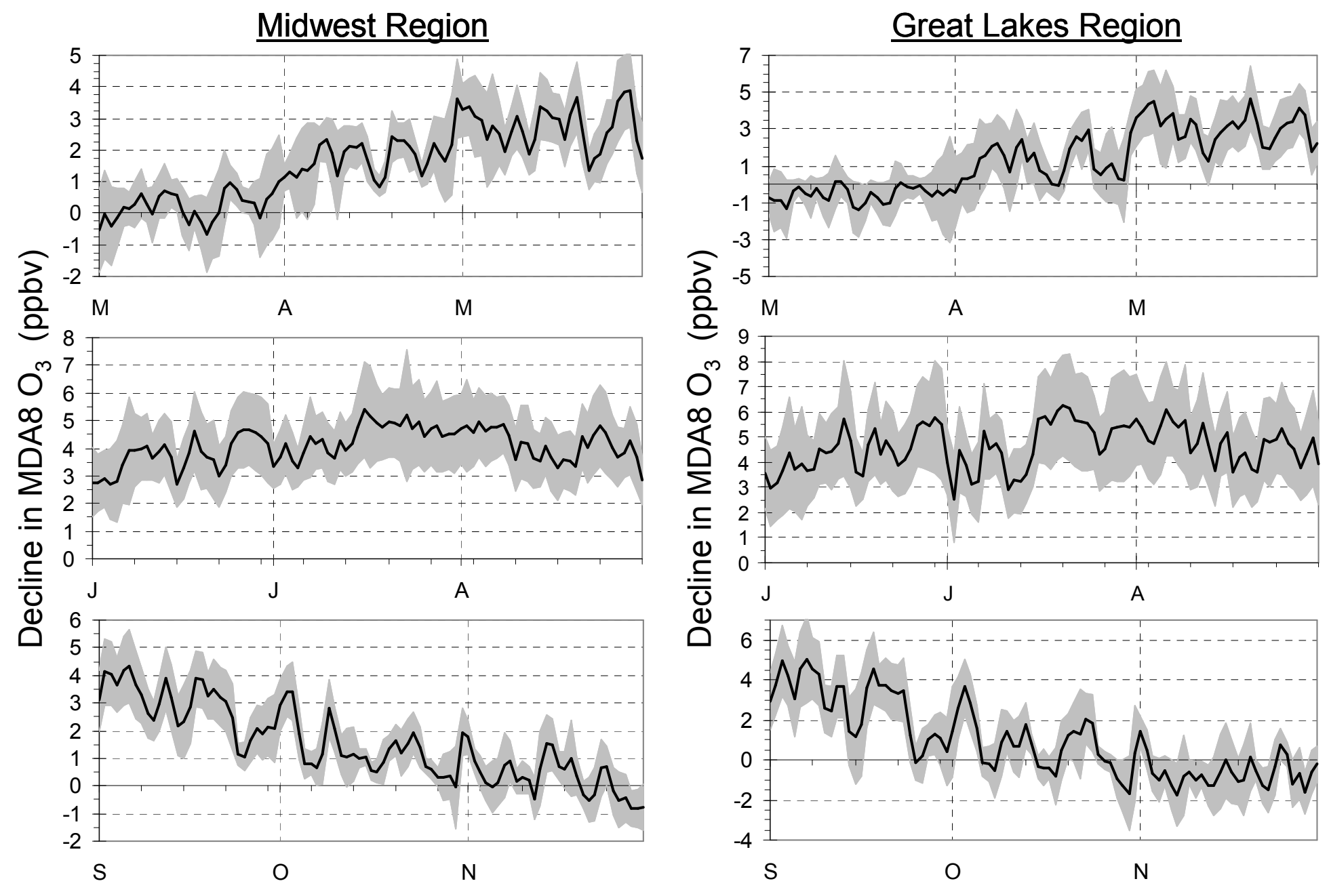

Figure A10. (cont'd) 

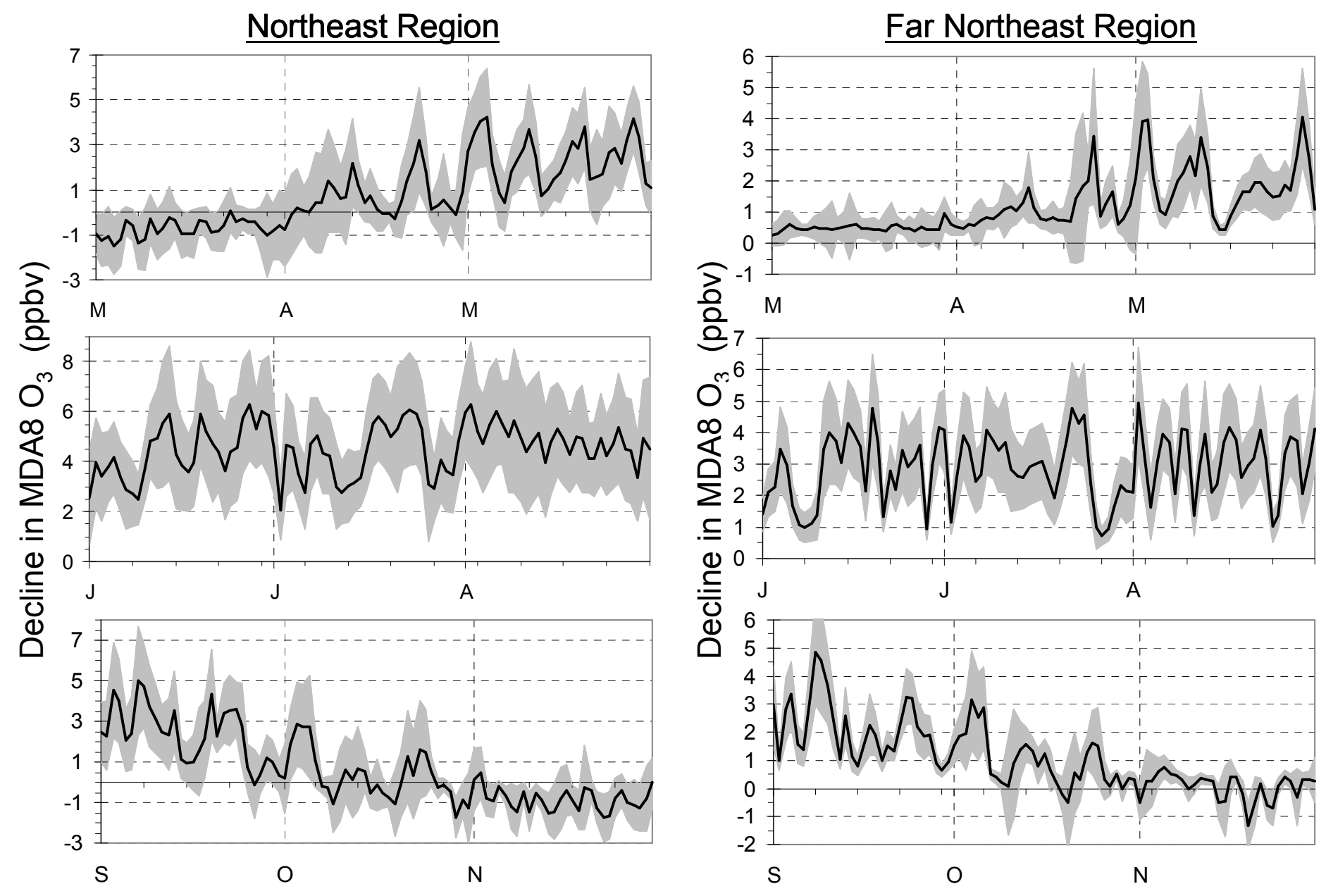

Figure A10. (cont'd) 


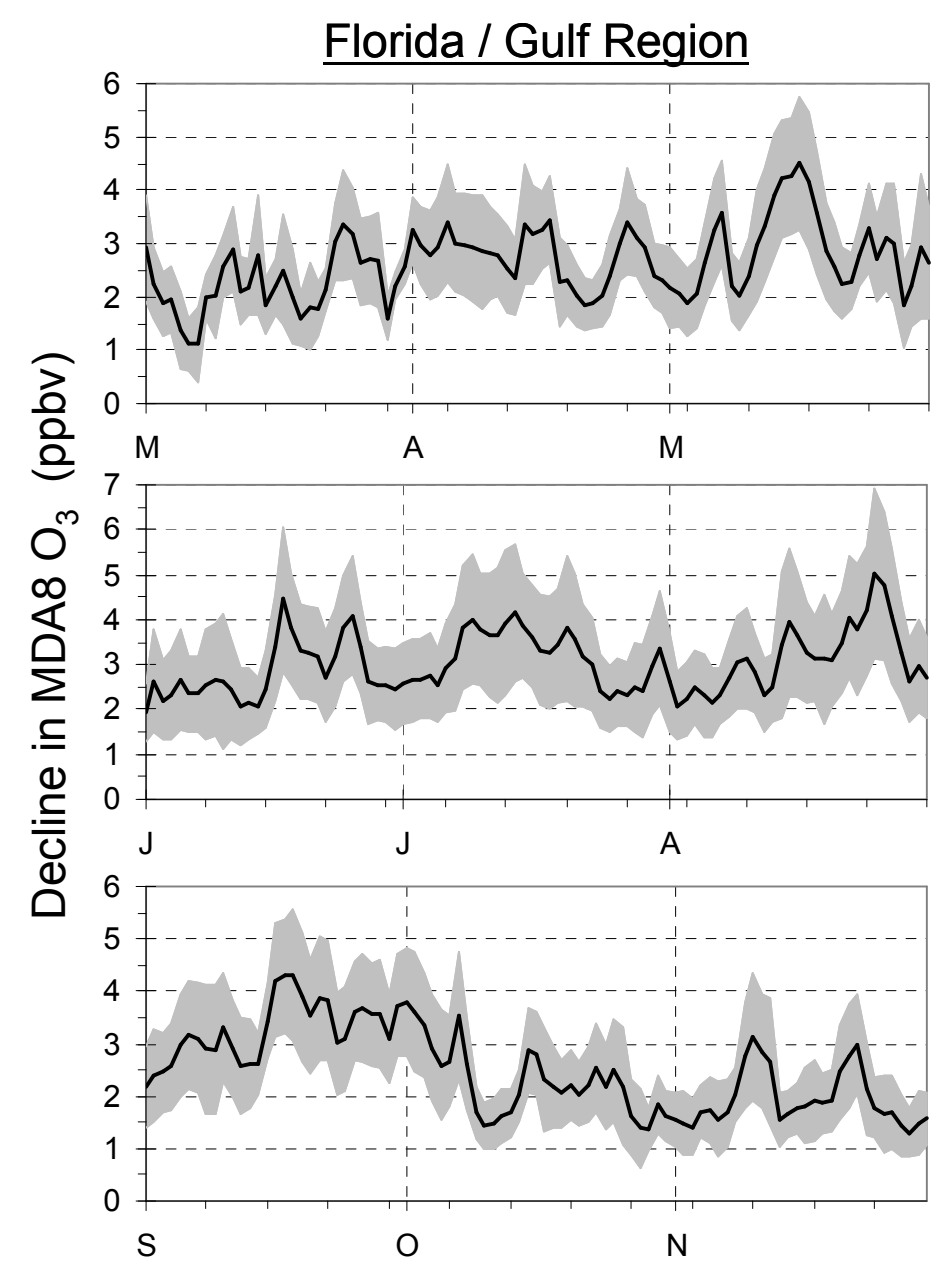

Figure A10. (cont'd) 